\title{
35. STELLAR CONSTITUTION (CONSTITUTION DES ÉTOILES)
}

\author{
PRESIDENT: L. Mestel \\ VICE-PRESIDENT: B. Paczynski
}

ORGANIZING COMMITTEE: A. N. Cox, P. R. Demarque, P. Giannone, C. Hayashi, I. Iben, R. Kippenhahn, A. G. Massevitch, G. Ruben, E. Schatzman, R. J. Tayler.

\section{INTRODUCTION}

The Commission's activities continue to expand and to invade other branches of Astronomy. Protagonists in the cosmological debate lean heavily on theoretical estimates of galactic evolution consequent on stellar evolution, and on constraints supplied by studies of nucleogenesis in stars. The solar neutrino problem is still with us, and is causing some to construct ever more exotic solar models. For others, the missing neutrinos and the $\mathrm{Li}-\mathrm{Be}$ problem are indications that our ideas on stellar hydrodynamics are inadequate. Observational work on the solar oblateness'-istimulated largely by rivalry between different relativistic theories of gravitation - has yielded as a bonus evidence that the sun is weakly pulsating: hopefully, this may turn out to be a valuable probe of the solar interior. Advances in dynamo theory and on the dynamical and thermal properties of rotating magnetic stars encourage a reappraisal of the observational data on the observably magnetic stars and on the role of magnetism in stellar interiors. Discovery of pulsars and compact X-ray sources has both supplied a laboratory for study of dense matter and general relativity, and has given an additional stimulus to work on close binary systems. And the growth in infra-red and millimetre astronomy offers hope that theories of star formation may soon be subject to observational check.

Conferences held since the last General Assembly include the Liège Colloquium on 'Stellar Hydrodynamics'; the Cambridge IAU Colloquium on 'Close Binary Systems'; the Vienna IAU Colloquium on 'Ap Stars'; and the Prague IAU Symposium on 'Basic Mechanisms of Solar Activity'. Some topics of interest to the Commission were discussed at the Trieste regional IAU meeting and at the Dallas Symposium on 'Relativistic Astrophysics'. A workshop on 'Convection' was held at Cambridge and on 'X-ray Sources' at Trieste. Scheduled meetings include the Geneva IAU Symposium on 'Star Formation' and the Nice IAU Colloquium on 'Convection'. A proposal has gone to the Executive for an IAU Colloquium on 'Nonperiodic Variable Stars' to be held at Bamberg in 1977.

Because of restrictions on space, this repört treats in depth just five subjects, selected because of their topicality: (1) Vital Statistics of Stars (Drs. Tinsley and Ostriker); (2) Stellar Convection (Dr. Gough); (3) Am and Ap Stars (The President); (4) Dynamical Nucleosynthesis (Di. Arnett); and (5) Neutron Matter and the Structure of Neutron Stars (Dr. Pines). Detailed references have had to be kept to a minimum. My thanks are due to my co-authors both for the excellence of their reviews and for their meeting the deadline. I am also grateful to Dr. Martin Schwarzschild for helpful advice on a number of occasions over the last three years.

2. VITAL STATISTICS OF STARS

(B. M. Tinsley and J. P. Ostriker)

\section{A. Summary}

Vital statistics of stars provide checks on the theory of stellar evolution and constraints on models for the evolution of stellar populations and their galactic environments. This report 
discusses the current status of several aspects of stellar statistics, but it makes no attempt to be a comprehensive or impartial review of literature in the field.

Early important studies of vital statistics of stars, in terms of stellar and galactic evolution, include those of Salpeter, Sandage, Schwarzschild, and especially Schmidt. Subsequent developments are reviewed in a number of recent papers, such as those of Audouze and Tinsley (Ann. Rev. Astr. Astrophys. 1976); Iben (Ann. Rev. 5, 571, 1967; 12, 215, 1974); Tammann (in Supernovae and Supernova Remnants, ed. Cosmovici, 1974); Tinsley (Publ. Astron. Soc. Pacific 1975); Trimble (Rev. Mod. Phys. 47, 877, 1975); and several contributions to IAU Colloquium 17 (Stellar Ages, eds. Delplace and Cayrel de Strobel, 1972); these reviews contain many more details and references than can be mentioned here, and cover several relevant topics that are omitted from this report.

A number of interesting conclusions can be drawn from available stellar statistics, with a minimal input from theory. They include the following, in order of increasing uncertainty.

(1) Most of a star's lifetime is spent on the main sequence.

(2) Most of a star's nuclear burning occurs in later stages of evolution.

(3) Substantial mass loss ( $\gtrsim 10 \%$ ) occurs for most disk stars.

(4) Low-mass stars leave white dwarf remnants, probably composed of carbon and oxygen.

(5) High-mass stars leave condensed remnants.

(6) The division occurs somewhere in the range $3-8 M_{\odot}$.

(7) The stellar birthrate is a decreasing function of stellar mass $(m)$ and time $(t)$, which can be represented in a useful approximation by letting $m^{-x} e^{-t / T}$ be proportional to the number of stars born in a unit logarithmic mass interval and unit time, where $x$ and $T$ depend slowly on mass and/or time. In the solar neighbourhood, $x \approx 0.25$ for stars near $0.5 / M_{\odot}$ increasing to $\approx 2.5$ for stars near $2 M_{\odot}$, and $T \gtrsim 5 \times 10^{9} \mathrm{yr}$.

(8) Stellar populations in external galaxies are consistent with points (1)-(7), except that the time-scale $(T)$ for star formation increases along the Hubble sequence from elliptical to Magellanic irregular types of galaxies.

We summarize below the statistical evidence supporting these statements, in varying amounts of detail and in a different order. Further constraints on stellar births, deaths, and evolution can be derived from the chemical composition of stars and the interstellar medium, but such data will not be considered here. Suffice it to note that some explanations of the distribution of stellar metallicities in the solar neighborhood require the initial mass function $\left(\mathrm{m}^{-x}\right)$ at early epochs to have been richer in massive stars than the function mentioned in (7).

\section{B. Birthrates in the Solar Neighborhood}

It is convenient to separate as far as possible our discussions of the initial mass function (IMF, which is the distribution of stellar masses at birth and integrates to unity) and of the time-dependence of the total birthrate, $b(t)$. This division is of course unsound in theory, since we have no reason to assume that the IMF has been constant, and not fully possible in practice, because of the finite lifetimes of stars that enter the statistics, but it is largely justified by the quality of information available.

\section{The IMF}

Stars with lifetimes shorter than the time-scale for long-term changes in the birthrate (averaged over a galactic rotation period), say with masses $z 2 M_{\odot}$, are represented in the main-sequence luminosity function in numbers proportional to the IMF, their main-sequence lifetimes, and the present birthrate $b\left(t_{1}\right)$. Thus the relative IMF for these stars can be derived directly from star counts (e.g., Schmidt; Ostriker $e t$ al.). Uncertainties enter chiefly through the conversion of spectral types of $O B$ stars to masses, and the possibility that a substantial fraction of massive stars are permanently hidden in dark clouds (Strom et al.).

Stars with lifetimes longer than the age of the Galaxy (masses $\leqslant 1 M_{\odot}$ ) are present in numbers proportional to the integrated past birthrate, $t_{1} b\left(t_{1}\right)$, so one can derive the average relative IMF 
for these stars, and scale this part of the IMF to that for massive stars, only by means of an estimate of $t_{1} b\left(t_{1}\right) / b\left(t_{1}\right)$ and assumptions regarding any variation of the lower IMF. Assuming an invariant IMF, it is possible to join the two pieces smoothly (thereby also estimating the IMF for stars of $1-2 M_{\odot}$ ) if the average past birthrate is rather close to the present rate; for example, the upper and lower IMFs illustrated by Audouze and Tinsley (1976, Fig. 1) can be joined to give a monotonic function only if $t_{1} \overrightarrow{b\left(t_{1}\right)} / b\left(t_{1}\right)$ is between about 8 and $20 \times 10^{9} \mathrm{yr}$. Of course this constraint may be violated if the birthrate for low-mass stars was relatively greater in the past, as has been proposed to account for the rapid initial enrichment of the disk (e.g., Schmidt, Truran and Cameron, Biermann and Tinsley), but implausibly discontinuous changes are needed near $1 M_{\odot}$ if changes of the IMF are the main cause of the initial enrichment and if the present smooth luminosity function is to be accounted for (Thuan et al.); one suspects that nature does not produce an IMF with a discontinuity that happens to lie just at the mass with a main-sequence lifetime equal to the age of the galaxy, but that other interpretations are called for (cf. Ostriker and Thuan, and the review by Audouze and Tinsley 1976).

There are still some uncertainties in the main-sequence luminosity function that gives the average IMF below $1 M_{\odot}$. For example, several surveys of $M$ dwarfs in the past few years raised the possibility of a much greater density, steeper luminosity function, and smaller scale-height than those given by conventional surveys. However, the most comprehensive of such surveys appear to have been dominated by systematic errors, which, when corrected, will restore the earlier rather flat luminosity function, typical old-disk scale height, and conventional density for $M$ dwarfs (cf. Weistrop, Faber et al.).

Salpeter originally fitted a single power law to the whole IMF, such that the number of stars born in a unit interval of $\log$ mass varied as $m^{-x}$, with $x=1.35$. The current estimates cited above point to a significantly flatter IMF for moderately low-mass stars $(x \approx 0.25$ for $0.4 \leqslant M / M_{\odot}<1.0$, but $x \approx 1.3$ for $\left.M \approx 0.4 M_{\odot}\right)$, and a steeper IMF for massive stars $(x \approx 2.2$ for $2 \lesssim M / M_{\odot} \lesssim 20$ ). Systematic errors enter a number of statistical calculations if the slope of 1.35 is adopted for all masses.

For the total birthrate, which is needed for example in estimates of the rate at which interstellar gas is being consumed, it is necessary to know the mass fraction of the IMF in stars below $0.2 M_{\odot}$ that are not significantly represented in any surveys to date. Weistrop's extensive star counts show that there is not a startling increase in slope of the IMF for very red dwarfs (Faber et $a l$.), but still a large mass could be hidden if the process of star formation involves fragmentation into masses too small to become main-sequence stars. An estimate of the lower IMF such as that given in figure 1 of Audouze and Tinsley (1976) can be extrapolated until the total mass per $\mathrm{pc}^{2}$ (including stars and interstellar gas) agrees with the dynamical density (Oort): the inferred lower mass limit is about $0.08 M_{\odot}$. This estimate neglects the mass of dead stars (white dwarfs etc.), which, depending on details of the past evolution and the remnant masses left by various stars, could be a substantial fraction of the unseen mass (cf., models referred to above), so that the effective lower stellar mass limit may be near $0.2 M_{\odot}$. If it is now assumed that the IMF has been constant, and that $b(t)$ has changed by a factor between say 1 and 3 during a galactic lifetime of $12-15$ billion years, the present total birthrate is found to lie between $3 \times 10^{-9}$ and $9 \times 10^{-9} M_{\odot} \mathrm{pc}^{-2} \mathrm{y}^{-1}$; a fraction between 0.7 and 0.9 of that amount is going into stars below $1 M_{\odot}$.

\section{The Birthrate $b(t)$}

The history of the birthrate, $b(t)$, in the solar neighborhood remains poorly known, because each of the empirical lines of evidence is subject to an indirect interpretation that involves many uncertain parameters of alternative evolutionary models. For example, the above constraint on $\overline{b\left(t_{1}\right)} / b\left(t_{1}\right)$ holds only if the IMF is assumed to have been smooth and not to have varied drastically near $1 M_{\odot}$.

In principle, the most direct way to determine $b(t)$ is to obtain stellar age distributions. The positions of various samples of $A-G$ evolving dwarfs and subgiants in the $H-R$ diagram are consistent with $b(t)$ decreasing by a very uncertain factor $\sim 5$ in the past $8 \times 10^{9}$ yr (Cayrel de Strobel, Clegg and Bell, Tinsley). If the factor mentioned lies between 1 and 7 , say, and if $b(t)$ 
is represented by an exponential decline, then the quantity $t_{1} \overline{b\left(t_{1}\right)} / b\left(t_{1}\right)$ is consistent with the above limits for a smooth IMF, if $t_{1} \leqslant 20 \times 10^{9} \mathrm{yr}$.

Some rather loose constraints on $b(t)$, the age $t_{1}$, and even variations in the IMF, can be derived from the abundances of radioactive isotopes in the early solar system: most likely, the IMF has not varied enormously (Reeves and Johns), $t_{1} \leq 6 \times 10^{9} \mathrm{yr}$, and the production of $r$-process elements was not extremely concentrated to early times (Schramm); but firm limits cannot be set, and no upper limit to $t_{1}$ can be derived, because of the many unknown parameters that affect the relations between $b(t)$ and the isotopic abundance ratios (Fowler, Tinsley).

If the strengths of CaII emission lines and/or space velocities of $\mathrm{K}$ dwarfs could be better calibrated in terms of age, they might give useful information on $b(t)$ for such stars (Wielen). The statistics of white dwarfs, discussed below, confirm the foregoing pieces of evidence that the birthrate has not been decreasing rapidly.

Further possible constraints on the behaviour of $b(t)$ can be set in the context of models for chemical evolution (cf. Audouze and Tinsley 1976), but as always there are too many parameters to allow a unique solution.

\section{Death Rates in the Solar Neighborhood}

Stellar death rates obviously reflect birthrates at various times in the past, the lag increasing with decreasing stellar mass, so in principle death rates provide further independ ent constraints on the IMF and on $b(t)$ at all past times. In practice, however, the original masses of dying stars and remnants are very difficult to identify, and prior knowledge of the birthrates is joined with mortality rates to provide astrophysical information on how various stars die. Let us assume that non-interacting stars with (original) masses up to $m_{w}$ die as white dwarfs (WD) after mass loss via stellar winds and/or planetary nebulae, and that stars with original masses greater than $m_{s}$ die as neutron stars (or black holes) after mass loss via stellar winds and a supernova (SN) explosion. Clearly, $m_{w} \leqslant m_{s}$. There may, a priori, be a mass interval between $m_{w}$ and $m_{s}$ in which carbon ignition occurs in a degenerate core, resulting in detonation and ejection of the core mass as iron (Arnett), leaving no remnant; but if the interval is wider than $\sim 0.5 M_{\odot}$, an excessive amount of iron is likely to be ejected (Ostriker et al., Arnett).

\section{White Dwarfs}

Since degenerate dwarfs arise from the least massive stars that die in the lifetime of the galaxy ( $\lesssim 1 M_{\odot}$ ) up to $\sim 4 M_{\odot}$ (cf. below), their present formation rate is insensitive to the value of $m_{w}$ but sensitive to the birthrate in the distant past. The present number ( $x$ cooling rate) is equal to the current birthrate lagged by the main sequence lifetime of the progenitors plus the white dwarf cooling time. Empirical estimates of the WD formation rate must rely on theoretical cooling rates; recent progress has reconciled the observed shape of the WD luminosity function with the temperature-dependence of cooling rates, assuming that the WD formation rate has been roughly constant and that WD are composed of carbon and oxygen (Sweeney; Lamb and Van Horn). A local formation rate $\sim 1.5 \times 10^{-9} \mathrm{WD} \mathrm{pc}^{-2} \mathrm{yr}^{-1}$ is indicated. Allowing for a factor 2 uncertainty in this rate, it agrees with the death rate of stars between 1 and any value of $m_{w} \gtrsim 3 M_{\odot}$, according to the birthrate discussed above. The WD statistics and theoretical cooling rates are not consistent with a rapidly declining stellar birthrate, or with a heavier-element composition (such as iron).

Methods of lestimating $m_{w}$, reviewed by Tinsley (1975), are all consistent with a value between 4 and $6 M_{\odot}$. To summarize briefly: (1) The existence of WD in the Hyades and Praesepe clusters shows that their precursor masses were greater than the cluster turnoff masses, i.e., $m_{w} \geq 2 M_{\odot}$. The probable membership of the WD LB1497 in the Pleiades cluster (Herbig and Luyten, Trimble and Greenstein, Jones, Greenstein) points to a much more interesting limit, $m_{w} \gtrless 6 M_{\odot}$ (2) The number of WD in the Hyades corresponds to the probable number of stars that were on the main sequence between the present turnoff mass and a limit $m_{w} \sim 3-5 M_{\odot}$ (van den Heuvel). (3) The ages of WD in clusters, determined from their cooling 
lifetimes, give estimates of their precursor main-sequence lifetimes relative to the cluster age, and hence estimates of $m_{w}$; values are up to 3-5 $M_{\odot}$ for the Hyades and 5-8 $M_{\odot}$ for the Pleiades (Sweeney). (4) Finally, $m_{w}$ can be derived from mass loss arguments: the condition for a star not to die explosively (by carbon detonation or by fuel exhaustion at a later stage) is that it loses mass to less than the Chandrasekhar limit of $1.4 M_{\odot}$ before carbon ignition. Empirical and semi-theoretical estimates of mass loss rates suggest that the most massive star which can achieve enough loss, i.e., the value of $m_{w}$ is 4 to $6 M_{\odot}$ (Fusi-Pecci and Renzini, Mengel, Scalo).

\section{Planetary Nebulae}

The stellar cores of planetary nebulae are generally assumed to be stars evolving from the red giant branch towards WD, so their formation rate should agree with that of WD. The statistics of planetary nebulae in the solar neighborhood given by Alloin et al. lead to a formation rate of a few times $10^{-10} \mathrm{pc}^{-2} \mathrm{yr}^{-1}$, which adequately agrees with the rate for WD quoted above. These authors also cite evidence that halo stars have a smaller probability than disk stars of forming detectable planetary nebulae, which implies that in order to use planetaries to trace the extent or mass of the galactic halo, a separate calibration is necessary.

\section{Supernovae}

Since the statistics of SN have been reviewed recently (Tammann 1974, Tinsley 1975), only a brief summary will be given here.

In the solar neighborhood, the rate of formation of radio $S N$ remnants is a few $10^{-11} \mathrm{pc}^{-2} \mathrm{yr}^{-1}$, which corresponds to the birthrate of stars above about 3 to $8 M_{\odot}$, yielding the estimate $m_{w} \sim 3-8 M_{\odot}$. It is difficult to derive a more precise local SN rate, because of the small numbers of historical SN, and theoretical ambiguities in the ages and distances of radio remnants. One can compare the local IMF with SN rates in external galaxies similar to the galaxy, but since the comparison is with SN rates per unit luminosity, the uncertainty in the extragalactic distance scale enters squared, precluding a useful estimate of $m_{s}$.

\section{Pulsars}

It appears to be impossible to determine the pulsar formation rate in the solar neighborhood within better than a factor of 10 , because of uncertainties in their distances, beaming effects, and ages (Arnett). The best available estimate is consistent with a pulsar formation rate equal to the SN remnant |formation rate, i.e., allowing all stars above about 4 or $8 M_{\odot}$ to become pulsars; but it is still consistent with a limited mass interval, say $4-8 M_{\odot}$ as suggested by Gunn and Ostriker, dying as pulsars, leaving the remnants of the most massive stars unknown. The scale height of the pulsar distribution is also consistent with these precursor mass estimates, but can neither help to establish them more firmly nor eliminate the possibility of a minority of pulsars originating from less massive stars. An important point is that the lower limit for pulsar formation is consistent with the upper limit $m_{w}$ for WD formation, so there is no difficulty in avoiding carbon detonation.

To conclude this section on local death rates, a very simple scenario seems to be consistent with many different types of evidence: as far as non-interacting stars are concerned, those with original masses less than $\sim 5 M_{\odot}$ die as white dwarfs after ejecting mass as stellar winds and planetary nebulae, while more massive stars die as neutron stars after mass loss and then a SN explosion.

\section{Lifetimes and Nuclear Burning on the Main Sequence and in Later Stages of Evolution}

For massive stars, the relative numbers on the main sequence (MS) and in later evolutionary stages give directly the relative times spent in each stage. Practical difficulties include identifying the masses of post-MS stars in the field, and of overcoming the statistics of small numbers in clusters. Early studies concluded from the luminosity functions of upper MS stars and 
supergiants that more massive stars spend most of their lives on the MS and burn most of their fuel there. The latter conclusion is particularly sensitive to the two problems mentioned, and a new investigation would be valuable.

There are two rather simple ways to see that most of the fuel of solar type stars is burned in post-main-sequence states. Theoretically we know that only $\sim 1 / 6$ of the mass of a $1 M_{\odot}$ star is burned before core contraction begins and the star leaves the main sequence. But, the dying stars of the same general type become white dwarfs of $0.6-0.8 M_{\odot}$ containing at least $5 / 6$ of the original mass. Since this cannot be hydrogen rich, or else the degenerate stars would explode, it follows that approximately four times as much fuel is burned after than on the main sequence. The observational argument (borrowing from Section V) is far simpler, being based on the fact that giants (of $\sim 1 M_{\odot}$ ) dominate the light from the disk population of M31 (inner disk - cf., Baldwin, Danziger, Fogel and Perssen) indicating that each star emits more light as a giant than as a dwarf.

For low-mass stars $\left(1-2 M_{\odot}\right)$, statistics cannot give MS lifetimes without a knowledge of the past birthrate; theoretical lifetimes have been used above to estimate $b(t)$ and the IMF. However, relative lifetimes at post-MS stages of evolution can be derived from field giant luminosity functions, and in principle from old clusters, although here the small number problem is crippling (cf. the early papers cited above; Cannon; Tinsley and Gunn, and references therein). This problem is especially serious for calculations of fuel burning, because the number of giants of a given mass and in a given magnitude interval (say), in a complete sample, is proportional to the time $\Delta t$ spent in that magnitude interval, while the nuclear fuel consumed is proportional to $L \Delta t$, where $L$ is the bolometric luminosity. Since the luminosity functions show that $\Delta t$ decreases by about a factor 2 per magnitude increase in $L$, very few stars may be present to represent energetically dominant stages of evolution. For example, Tinsley and Gunn find that the luminosity function of old-disk giants in the field is consistent with low-mass Population I stars having a helium core mass $\approx 0.4 M_{\odot}$ at core helium ignition, after about $8 \times 10^{8} \mathrm{yr}$ on the first giant branch (assuming the theoretical core mass $\approx 0.2 M_{\odot}$ at the end of MS evolution), spending $(9-16) \times 10^{7} \mathrm{yr}$ burning helium in the core as an early $\mathrm{K}$ 'clump' giant, and ending their lives with $L \approx 10^{4} L_{\odot}$ and a core mass $0.7 \pm 0.1 M_{\odot}$ after a further $(0.1-0.2) \times 10^{7} \mathrm{yr}$ on the second giant branch. Possibly a third of the nuclear fuel is burnt as a (bolometrically) very luminous $M$ giant, in stages that occupy only about $10^{-3}$ of the star's post-MS lifetime and about $10^{-4}$ of its total lifetime. It is encouraging that the final core mass derived this way agrees very well with the masses of white dwarfs (Trimble and Greenstein). The inadequacy of clusters as samples of evolving stars can be seen by noting that in M67, the giants present would lead one to conclude that low-mass giants end their lives with $L \sim 450 L_{\odot}$; the total number of additional giants needed to give M67 a luminosity function proportional to that of the field stars is only 0.7 stars!

\section{E. Birthrates Outside the Solar Neighborhood}

\section{The IMF}

Apart from counts of the brightest stars in other regions of our galaxy and the nearest external galaxies (van den Bergh), the IMF outside the solar neighborhood must be estimated indirectly. It is useful to adopt the working hypothesis that the local IMF is 'universal' as well as constant in time, and to check empirical evidence against this hypothesis, realizing that small and sometimes great departures will not be discernible.

Much effort has been devoted to population syntheses, predicting the colors, mass-to-light ratios, etc. of theoretical stellar populations with series of IMFs, and comparing these with observations. The results are consistent with our working hypothesis being true in 'typical' galaxies, but they by no means prove that there is a universal IMF - the predicted quantities are unfortunately sensitive to other parameters of the synthesis (metallicity, $b(t)$, etc.), and real galaxies show a scatter of properties that defies explanation with a very small number of model parameters (e.g., Tinsley, Searle et al., Larson and Tinsley, Sargent and Tinsley). More will be said about the average properties of galaxies in the next subsection. 
There is some evidence for an IMF enhanced in low-mass stars in the halo of our galaxy. Schmidt finds that stars with halo kinematics, passing through the solar neighborhood, have a rather steeper luminosity function for small masses $\left(\sim 0.2-0.6 M_{\odot}\right)$ than is found for the disk stars. If galaxies have invisible (to date) halos (Ostriker and Peebles), and if these are made of stars, the halo IMF must have been very different from the local function, either being weigh ted towards very low-mass stars, or being dominated by massive stars that have evolved to invisible remnants. We note that the depletion of the lower main sequence in globular clusters (van den Bergh) does not mean that their IMF was so biased, because low-mass cluster stars would probably have evaporated (Ostriker et al.) from these systems.

\section{The Birthrate $b(t)$}

Population syntheses that help to define the IMF in regions outside the solar neighborhood also set constraints on their birthrates. The studies referred to above show that the systematic changes in photometric properties and gas content along the Hubble sequence of galaxies is consistent with 'average' galaxies having the same IMF, and having the same age $\left(\sim 10^{10} \mathrm{yr}\right)$, but differing in the time-scale for converting gas to stars; the inferred time-scale, $T$, changes from $\sim 10^{9} \mathrm{yr}$ for elliptical galaxies, to $\sim 10^{10} \mathrm{yr}$ for the latest spirals and Magellanic irregulars. Unfortunately, factors that might imply considerable departures from this straightforward scheme cannot be disentangled, using the methods and data so far available. There are of course peculiar galaxies whose colors suggest extreme youth (Searle and Sargent, Searle et al.), others with evidence for recent outbursts of star formation (van den Bergh), etc., while even apparently normal galaxies show a considerable scatter in photometric and other properties at a given Hubble type. It is encouraging that variation of the time-scale for $b(t)$ may be responsible for the major trends in stellar populations among galaxy types; future studies of detailed differences and discrepancies might yield important information on the processes of formation and evolution of stars and galaxies. Dynamical and chemical aspects of galactic evolution cannot be separated from the understanding of stellar birthrates, but these topics are beyond the scope of this report - see, e.g., several papers in the IAU Symposium No. 58, and Audouze and Tinsley (1976).

\section{F. Death Rates Outside the Solar Neighborhood}

Apart from meager statistics on planetary nebulae in more distant regions of our galaxy and in some nearby systems (Alloin et al. and references therein), the only detectable stellar deaths outside the solar neighborhood are SN. Again, we briefly summarize the points made in recent reviews cited above.

The distribution of Type II SN in external galaxies strongly supports the view that their precursors are massive (OB) stars. The lack of known Type II SN in all by Sc and Sb galaxies, in spite of the obvious presence of $O B$ stars in earlier and later types, although indicative of the relative paucity of extreme Population I material in Sa systems, appears to be a quirk of small number statistics. If stellar birthrates are assumed to vary along the Hubble sequence according to the simple hypothesis discussed above, then the relative rates of Type II SN that are predicted from relations between stellar birthrates and galactic colors, agree with the observed relative rates (Sargent et al., Larson and Tinsley). It is intriguing that this agreement holds, at least for spiral galaxies, when all types of SN, including identified Type I's, are counted in the relative rates. Type I SN are conventionally supposed to arise from explosions of very old white dwarfs, single or in binary systems, but the only piece of statistical evidence strongly favoring this view is their occurrence in elliptical galaxies, where the stellar population is supposed to be all very old (cf., the reviews cited above) and in the outer parts of spiral galaxies. On the theoretical side, there are no convincing models either for the explosive death of a very old single WD, or for the accretion of enough matter on to a WD from a companion to cause a complete explosion rather than a series of surface outbursts (as a nova). It may therefore be useful to consider, in the future, alternative stellar precursors for Type I SN. 


\section{G. The Mass Balance in the Solar Neighborhood}

Vital statistics of stars can be used to estimate the time-scale for depletion of the interstellar medium (ISM) in a region. If the birthrates and death rates were accurately known, the statistics would give this time-scale independently of an evolutionary model. But since ideal data are not available, it is found that the statistical approach by itself is not very powerful. Consider, for example, the IMF and birthrate for the solar neighborhood, discussed above. From the mass distribution of stars entering the quoted estimates of the total birthrate, we find that dy ing stars are returning matter to the ISM at about $8 \times 10^{-10} M_{\odot} \mathrm{pc}^{-2} \mathrm{yr}^{-1}$, with about a $20 \%$ uncertainty arising from stars with masses $\sim 1$ to $2 M_{\odot}$ whose death rate depends significantly on the past $b(t)$. About $1 / 4$ of the return is from stars above $5 M_{\odot}$, i.e., the SN precursors. A similar estimate of the return rate is obtained directly from the SN and WD (or planetary nebula) formation rates, as must be the case since we found them to be in agreement with the death rates predicted from the birthrates; but, even if the death rates were statistically well known, they would not give a good estimate of the rate at which stars return matter to the ISM without a knowledge of the IMF, and of $b(t)$ for stars between 1 and $2 M_{\odot}$, which are needed to evaluate the average mass loss associated with each stellar death.

The above figures suggest a net gas consumption rate between 2 and $8 \times 10^{-9} M_{\odot} \mathrm{pc}^{-2} \mathrm{yr}^{-1}$, in the solar neighborhood. If the ISM (including neutral and ionized hydrogen, molecules, helium, etc.) has a density between 10 and $20 M_{\odot} p^{-2}$ the time-scale for its depletion into long-lived stars and remnants thus lies between $10^{9}$ and $10^{10}$ years indicating, independently, an $e$-folding rate for the gas comparable to that estimated for $b(t)$. Most of the uncertainty in this time-scale comes from the estimates of $t_{1}$ and $\overline{b\left(t_{1}\right) / b}\left(t_{1}\right)$ that were used above to derive the present birthrate of stars below $1 M_{\odot}$ from their total density; a constant IMF for such stars has been assumed.

Here we must mention the possibility that the mass budget of the ISM is affected by infalling gas (Oort, Larson). Current interpretations of the relevant data on high-velocity hydrogen are widely divergent (cf. Hulsbosch, Verschuur), but there seems to be no compelling argument against an infall rate $\sim 10^{-9} M_{\odot} \mathrm{pc}^{-2} \mathrm{yr}^{-1}$, and it is theoretically not implausible, either as relic material representing the last stages of formation of the galaxy (Gunn and Gott), or as gas lost from the envelopes of halo stars (Ostriker and Thuan). In view of this possibility, stellar birthrates and death rates cannot confidently be used to derive evolutionary timescales for the galaxy. A similar problem of course arises where there are likely to be galactic winds, e.g., in elliptical galaxies and the nuclear bulges of spirals (Mathews and Baker, Faber and Gallagher), for both the accumulation of ISM and star formation can be entirely inhibited in spite of a substantial rate of mass loss from dying stars.

Further constraints can be set on stellar birthrates, death rates, and their effects on galactic evolution when the statistical data are tied to considerations of dynamical and chemical evolution (cf., references cited above). In trying to make consistent and rather detailed interpretations of all the relevant types of data, one always meets the need for a theory of star formation. Vital statistics provide at best information on only a very narrow range of stellar masses and birth epochs, and we urgently need to be able to predict how birthrates, and especially the IMF, vary with the astrophysical environment. It is encouraging that already rather simple and persuasively consistent hypotheses can account, in broad outline, for the relations among stellar birthrates and death rates, and for the changes in the properties of stellar populations along the sequence of galaxy types. Many of these ideas were being explored already in the 1950's; while subsequent efforts have changed and filled in some of the details, much remains to be done, both observationally and theoretically, to develop the empirical approach to stellar and galactic evolution.

\section{H. Acknowledgements}

This work was supported in part by National Science Foundation grants MPS74-18970 and ATS75-16329 and by the Alfred P. Sloan Foundation (B.T.). 


\section{STELLAR CONVECTION \\ (D. O. Gough)}

The most important function of a convection theory for stellar model building is to determine the temperature stratification in terms of the heat flux. Apart from it some recent work by Latour et al., mixing length theory, in one or other of its guises, still provides the only prescription that is used. Unfortunately this is not a reliable procedure because of the crude way in which the dynamics is treated. Furthermore, the resulting formulae depend on the mixing length $l$ which occurs in the theory as an undetermined function. Work on convection that may one day lead to a more satisfactory theory is taking place, but none of it has yet reached the point to warrant displacing the methods currently practised in stellar evolution computations. The reader is referred to the reviews by Spiegel (Ann. Rev. Astr. Astrophys. 9, $323,1971 ; 10,261,1972)$ for a discussion of the astrophysically relevant work on convection up to 1972 .

\section{A. Attempts to Model Thermal Convection}

One of the principal factors inhibiting progress in stellar convection theory is that conditions in stars are very different from those in the laboratory. Stellar convection is characterized by high values $\left(10^{20}\right)$ of the Rayleigh number $R$, which is a dimensionless measure of the temperature gradient, and low values $\left(10^{-9}\right)$ of the Prandtl number $\sigma$, which is the ratio of kinematic viscosity to thermal diffusivity. On the other hand in the laboratory $R$ is quite low $\left(<10^{11}\right)$ by astrophysical standards, and $\sigma$ is of order or greater than unity. Moreover, stellar convection zones extend typically over many scale heights of pressure and density, leading to compressible motions, whereas in the laboratory the depth of a convecting layer is always a minute fraction of a scale height: the motion is essentially incompressible and can be described by the Boussinesq approximation.

Most of the theoretical work is aimed at mimicking laboratory conditions. A thin layer of fluid bounded by two isothermal planes, the lower boundary being at the higher temperature, is usually considered. The equations of motion are solved, usually in the Boussinesq approximation, either numerically at moderate $R$ and $\sigma$ or analytically at low $R$ close to the critical value $R_{c}$ at which a static fluid layer becomes unstable to convection. At present the computational difficulties are too severe to extend these calculations to values of $R$ and $\sigma$ of astrophysical interest. The main objective is to determine the dependence of the Nusselt number $N$, a dimensionless measure of the heat flux, on $\sigma$ and $R$. A plausible extrapolation procedure might then lead to a better prescription for stellar convection.

The most obvious procedure, one might think, would be to apply mixing length theory to laboratory convection and attempt to determine $l$ experimentally. However it is the astronomer's belief that this is of no use, for whereas in the laboratory eddies extend across the whole of the convecting layer, in a compressible fluid many scale heights deep the shear produced by differential expansion and contraction of vertically moving fluid is thought to disrupt the convective motion in about a scale height (Schwarzschild). This is a nonlinear argument, and so is not contradicted by the fact that the most unstable linear modes extend across the entire convectively unstable region (Spiegel, Böhm). Accordingly, $l$ is presumed to be proportional to a density or pressure scale height (Opik, Vitense), usually the latter, the constant of proportionality being determined astronomically, and contact with terrestrial experience is lost.

In its most usual form the mixing length theory provides a local relationship between the heat flux $F$ and the superadiabatic temperature gradient $D \equiv \nabla-\nabla_{\mathrm{ad}}$. There are many uncertainties in the theory, and consequently there is opportunity to incorporate into it several adjustable parameters, though only two are of immediate interest: $\alpha=l / H$, where $H$ is an appropriate scale height, and a measure $\gamma$ of the radiative losses. The theories are calibrated either by constructing a solar model and adjusting it to have the correct luminosity and effective temperature at an age of about $4.7 \times 10^{9} \mathrm{yr}$ (Schwarzschild et al; Sears), or by fitting a 
theoretical main sequence to a young cluster diagram (Demarque and Larson; Copeland, Jensen and Jørgensen). Both methods yield $\alpha \simeq 1$, the precise value depending on the details of the theory adopted, but leave $\gamma$ undetermined. Some comfort is derived from the observation that this implies an eddy size at the top of the solar convection zone comparable with the length scale of the granulation (Schwarzschild). The gross structure of a main sequence stellar model is insensitive to $\gamma$, which matters only near the outer edge of the envelope convection zone. The parameter $\gamma$ does affect the convective envelopes of red giants, however, which have large nonadiabatic regions (Henyey et al; Schwarzschild). Red giant models are probably more sensitive to other details of how convection is treated, too, especially in the surface layers where fluctuations are large in magnitude and horizontal extent.

It should be noticed that the mixing length formalisms used in stellar structure computations are based on the Boussinesq approximation to incompressible flow. One would have expected this to have been valid had $l$ been much less than $H$, but indications are that it is not a good approximation otherwise (Graham, Deupree). Thus the calibration $l \simeq H$ exposes an inconsistency in the theory. However, the evidence for the functional dependence $l \propto H$ is hardly overwhelming, and adopting it no doubt introduces errors that are just as great. The apparent success of the mixing length formalism lies in the fact that the gross structure of a main sequence stellar model is almost independent of functional form of the relationship between $F$ and $D$ (Gough and Weiss).

Stellar mixing length theory ignores viscosity. This sounds plausible since the Reynolds number of the heat-transporting flow is large and $\sigma$ is small. It implies that $N$ depends on $\sigma$ and $R$ only in the combination $\sigma R$ which is independent of viscosity. Furthermore, since $N$ increases with $R$ at fixed $\sigma$, it must therefore increase with $\sigma$ at fixed $R$, provided $\sigma$ remains small.

The beginnings of an attempt to bridge the gap between laboratory and stellar conditions, using a truncated modal expansion, has recently been reported (Gough, Spiegel, Toomre). Although the analysis is in the Boussinesq approximation, it can treat with the same assumption the extreme values of $R$ and $\sigma$ typical of stars and the more moderate values encountered in the laboratory. The procedure can be made to reproduce some of the features of laboratory convection, but its most obvious drawback is that it contains several undetermined parameters. In its simplest form there are just two such parameters, characterizing the horizontal scale and shape of the convective eddies. Although this is perhaps an improvement over mixing length theory, which depends on an undetermined function $l$, an unambiguous calibration by comparison with laboratory convection has not been possible. The theory has the property that for $\sigma \ll 1, N$ is a function of $\sigma R$, provided the convection is three-dimensional, which accords with astronomers' prejudices.

It should be pointed out, however, that the $\sigma$ dependence of $N$ is not universally believed. This arises partly because almost all laboratory experience is with fluids that have $\sigma \gtrless 1$, and for these both theory and experiment show that $N$ is almost independent of $\sigma$ at fixed $R$. Furthermore, numerical solutions of the Boussinesq equations at moderate $R$, which until recently have always constrained the flow to be two-dimensional, have predicted almost no $\sigma$ dependence, and even a slight increase of $N$ as $\sigma$ is decreased below unity (Veronis; Quon; Moore and Weiss), though it has been argued that this may be a result of constraining the horizontal length scale of the motion (Lipps and Somerville; Willis, Deardorff and Somerville). The simplest modal analysis predicts that $N$ is independent of $\sigma$ when the motion is twodimensional. Analytical expansions of the full Boussinesq equations for $R$ near $R_{c}$ reveal only a weak dependence on $\sigma$ in that case too (Schlüter, Lortz and Busse) but, like the modal results, suggest a strong decrease in $N$ at low $\sigma$ when the motion is three-dimensional. This led Jones, Moore and Weiss to investigate numerically axisymmetrical convection in a cylinder which, though mathematically dependent on only two space variables, is geometrically threedimensional. They reproduced the analytical results for $R$ just above $R_{c}$, but showed that at moderate $R$ the flow readjusted itself to resemble the two-dimensional flows, and produced an $N$ that is independent of $\sigma$ at high and low $\sigma$, and slightly decreasing with $\sigma$ in the neighbourhood of $\sigma=1$. The issue is unresolved. Jones et al. suggest that their flow is unstable and that at sufficiently high $R$ it would become turbulent with $N$ independent of viscosity at 
low $\sigma$. Moreover, three-dimensional calculations reported recently by Veltishchev and Żelnin at $\sigma=0.7$ and $\sigma=1$ suggest that the flow does not adjust itself to the kind of structure that is preferred when axisymmetry is imposed, and that $N$ is less when $\sigma=0.7$ than it is when $\sigma=1$ : This concurs with the evidence from laboratory experiments, though this is admittedly weak. Finally, if a convection theory based on eddies of scale $l \propto H$ and governed by dynamics similar to that exhibited by the two-dimensional and axisymmetrical Boussinesq computations (and therefore implying $N$ is independent of $\sigma$ ) were subject to the usual astronomical calibration, the result would be that $l$ would be but a very small faction of $H$, which most astronomers would find unpalatable.

Little study has been made of fully developed convection in a layer of compressible fluid many scale heights deep. Graham has made some two-dimensional computations for a perfect gas at moderate $R$ and $\sigma$. The property exhibited by similar Boussinesq calculations that $N$ is a decreasing function of $\sigma$ when $\sigma \simeq 1$ is accentuated as the layer depth, and the effects of compressibility, are increased. Moreover, no tendency for eddies to break up on a scale of $H$ was found. Graham's more recent three-dimensional compressible calculations yield similar results. Compressible modal calculations have also been performed in the anelastic approximation; by van der Borght with $\sigma=1$ and by Latour et al. under more realistic stellar conditions modelling an A star envelope. As with the Boussinesq calculations there are undetermined parameters which can be chosen to produce plausible results. Once again, time-dependent calculations show no tendency for the motion to break up into eddies on the scale of $H$.

\section{B. Penetration and Overshooting}

The edges of stellar convection zones are not rigid inpenetrable boundaries as they are in most laboratory and theoretical investigations. The density stratification changes from being convectively unstable to convectively stable, from the point of view of linear stability analysis, and fluid accelerated in the convectively unstable region can penetrate, or overshoot, into the adjacent stable regions.

This phenomenon has been of interest particularly to meteorologists interested in mixing at the atmospheric inversion. D. W. Moore (IAU Symp. 28) gives a brief account of the relevant physics. A convective element, or thermal, on reaching the top (or bottom) of the convectively unstable region, still has a temperature excess (or deficiency) relative to its immediate surroundings and continues to experience a buoyancy force. If the element were to maintain its identity and move adiabatically in pressure equilibrium with its surroundings, buoyancy would not disappear until the level $z=z_{s}$ were reached at which the specific entropy were the same as at the level at which the element originated. This point does not necessarily mark the edge of the zone of penetration, however, because the element still has momentum to carry it on yet further. Entrainment of stable fluid, on the other hand, retards the motion of the element. Thus $z=z_{s}$ may either overestimate or underestimate the extent of penetration. Observations of the motions of cloud tops suggest that it is usually an overestimate, though in some circumstances, such as in tropical storms, large plume-like structures penetrate well above the tropopause. An additional complication, usually ignored by meteorologists in this context, is radiative diffusion, which tends to reduce both the buoyant acceleration and retardation by reducing temperature fluctuations. Buoyant thermals penetrating into the stable layer advect heat upwards, though this is offset by the induced return flow. Near the outer edge of the penetrated region both upward and downward moving fluid presumably transport heat counter to the net flux.

Some aspects of the situation can be modelled with the ice-water experiment. This consists of a layer of water cooled from below at $0^{\circ} \mathrm{C}$ and with its upperiboundary maintained above $4^{\circ} \mathrm{C}$, the temperature of the density maximum. Laboratory experiments show that the unstable layer extends beyond the limits it would have occupied had there been no motion (Townsend, Adrian), and in addition plume-like motions in the unstable region penetrate into the stable layer above. Adjacent layers of convectively stable and unstable fluid have also been created by inducing spatially varying temperature gradients in water near room temperature, either by 
imposing time varying boundary conditions (Krishnamurti; Deardorff, Willis and Lilly) or by internal heating or cooling (Faller and Kaylor; Whitehead and Chen). The nature of the motions in the stable layer is not entirely clear, but the temperature fluctuations observed by Townsend seem to be the product of trapped gravity waves. Theoretical numerical experiments in two-dimensions by Moore and Weiss also exhibit the encroachment of the unstable region into the region that would have been stable in the absence of motion, and the excitation of gravity waves. They also predict weak viscously driven countercells, which are not seen in the laboratory, and little evidence of plumes. Earlier steady one-mode mean-field calculations, which in some sense represent two-dimensional motion, yielded similar results, without the gravity waves (Musman). Thus some of the observed features of laboratory experiments are reproduced theoretically; the differences, as Spiegel has pointed out, might result from the two-dimensional constraint imposed on the numerical computations.

Although the ice-water experiment sheds some light on the mechanism of penetration it seems difficult to generalize to stellar conditions. There is some evidence from the twodimensional numerical experiments that penetration increases as Prandtl number decreases (D. R. Moore). Modal calculations by Latour et al. modelling three-dimensional convection in A star envelopes predict greater penetration by the almost plume-like columns in the centres of hexagonal cells than by two-dimensional rolls. However, this analysis has not been applied to the ice-water problem; there is yet no bridge between stars and laboratory experience.

The various theoretical prescriptions that are usually employed to describe overshooting from stellar convection zones are all essentially based on mixing length theory (Spiegel, Parsons, Ulrich, Scalo, Shaviv and Salpeter). Of necessity they are nonlocal theories, though they all rely on the Boussinesq approximation. They have been used, in particular, to model the solar atmosphere which is perhaps the most sensitive astrophysical testing ground at present, because quite detailed comparison of theoretical predictions with observations can in principle be made. It is not easy to deduce the height dependence of the solar atmospheric velocity fluctuations, nor is it easy to disentangle convective motion from waves, though an attempt has been made (Frazier). It seems likely, however, that velocities of about $2 \mathrm{~km} \mathrm{~s}^{-1}$ extend well above the photosphere (de Jager), which agrees with a model computed by Ulrich, though the theory does appear to overestimate the overshoot. Travis and Matsushima, using a theory of Spiegel, compare their models with limb darkening measurements and conclude also that too great an overshoot is predicted if a mixing length to scale height ratio $\alpha$ of about unity is adopted; they favour $\alpha \leqslant 0.35$, in contradiction to the usual calibration. A subsequent investigation by Travis and Matsushima of the colours of cool main sequence stars and metal-deficient subdwarfs also suggester a low value for $\alpha$. Nordlund, using Ulrich's approach, found overshoot to a lesser degree for a given $\alpha$, and produced a model in better agreement with the Harvard-Smithsonian Reference Atmosphere and similar to an earlier model built, by Parsons using a convective heat flux calculated from a nonlocal estimate of vertical velocity and a local estimate of temperature fluctuations. In contradiction, Edmonds's analysis of the photospheric velocity and brightness fluctuations favours a greater degree of overshoot, so the matter seems unresolved. One thing that does seem clear is that at their present stage of sophistication nonlocal convection theories should not be relied upon to explain fine details, especially in regions in which the assumptions on which they are based are not satisfied. All the theories have adjustable parameters and can no doubt be tuned to rationalize the limb darkening; adjusting the radiative loss coefficient in Spiegel's theory, for example, could probably lead to an atmosphere hardly distinguishable from Nordlund's with an $\alpha$ consistent with the evolutionary calibration. Indeed Spruit has produced a model with the correct centre to limb flux variation using a local mixing length theory with no overshoot at all, though presumably this does not reproduce the fluctuation measurements discussed by Edmonds. Although there seems to be too much uncertainty in the theories at present to apply such subtle tests, detailed analyses of inhomogeneous atmospheres must eventually be undertaken both for theoretical model building and for analysing observational data. Horizontal temperature fluctuations increase the horizontally averaged opacity, for example, since opacity is a steeply increasing function of temperature, which leads to an increase in the actual mean temperature gradient. Furthermore, since the magnitude of the fluctuations decrease with height, the temperature gradient currently inferred from limb 
darkening observations is overestimated when fluctuations are ignored. Abundance measurements may be affected. Turbulent Reynolds stresses generated by both the convection and the gravity waves in the photospheric regions also influence the stratification.

Another important consequence of overshooting is material mixing, particularly at the edges of convective cores. Early estimates (Roxburgh; Saslaw and Schwarzschild) which ignored the influence of the convective energy flux on the temperature stratification, implied negligible mixing rates. But recently Shaviv and Salpeter pointed out that the modification to the stratification significantly increases the penetration of the motion into the stable envelope, just as in the case of the ice-water experiment. Maeder and Cogan independently confirmed this conclusion with more detailed calculations. The influence of the overshooting on colourmagnitude diagrams for old open clusters was subsequently investigated. Of particular interest is the position and magnitude of the gap at the top of the main sequence, which can be more accurately reproduced theoretically if an appropriate degree of mixing at the core boundary is assumed (Maeder, Prather and Demarque). Using Shaviv and Salpeter's prescription for overshooting, Maeder found once again that a value of $\alpha$ somewhat less than unity gives the best results. This too should not be regarded as contradicting the usual calibration, partly because the chemical composition adopted for the models may not have been appropriate, partly because there lies buried in the mixing length formalism an undetermined parameter in the relation between velocity and temperature fluctuations that does not appear in the formula for the heat flux, partly because the geometry of the core has not been taken into account, and partly because the ratio of the mixing length to pressure scale height can hardly be a universal constant.

Calculations by Sugimoto and Nomoto and Iben suggest that theoretical predictions of nucleosynthesis in post main sequence stars would be significantly affected by overshooting beneath convective envelopes. It would also have some bearing on the observed lithium abund ance in the sun (Spiegel).

\section{Subcritical Convection}

In the relatively straightforward case of ordinary convection discussed in $\S \mathrm{A}, N$ is an increasing function of $R$ at fixed $\sigma$. That is not necessarily the case when agents such as rotation, magnetic fields or nonuniformities in composition are present to inhibit the motion. The minimum Rayleigh number $R_{0}$ above which convection can exist is modified by the presence of the stabilizing agent, but it is not always possible to determine its value by linear stability analysis. It is often the case that direct convective motion of finite amplitude can adjust itself to reduce the efficacy of the stabilizing forces, and so exist at a Rayleigh number below the value $R_{c}$ predicted by linear theory (Veronis). This is called subcritical convection. Of course such a state can be achieved only if it were approached by lowering $R$ from a value greater than $R_{c}$ or if a metastable state, with $R_{0}<R<R_{c}$, were appropriately perturbed by a finite amount. It seems that the latter is often achieved spontaneously because in many circumstances there is a range of $R$ below $R_{c}$ within which the fluid is overstable, that is to say unstable to infinitesimal oscillations (Veronis, Weiss). Weak experimental evidence exists to support the idea that such motion might grow to an amplitude great enough to trigger subcritical convection (Turner, Shirtcliffe, Rossby).

The most widely studied problem of this type, and perhaps the easiest to understand, is thermohaline convection. Turner (Buoyancy effects in fluids, 1973) summarizes well the present state of knowledge. The case of interest here is when salt stabilizes a layer of water heated from below. Veronis studied the overstability and subcritical direct convection and gave a simple physical explanation of why they should occur. Laboratory experiments reveal that instability does occur first as a growing oscillation, and that convection subsequently organizes itself into a series of superposed shallow layers separated by diffusive interfaces (Turner and Stommel), a configuration that has been observed to occur naturally (Hoare; Neal, Neshyba and Denner). The fluxes of heat and salt appear to be controlled by the diffusive interfaces, and Turner has observed that their ratio $\Phi$, when measured in units of the fluxes that would have occurred had motion been absent, appears to be independent of the ratio $\lambda$ of the jumps in salinity and 
temperature across the layer, over a wide range of $\lambda$. Indeed, it has been suggested (Turner) that this value of $\Phi$ depends only on the diffusion coefficients of the fluid, though recent experimental work indicates that it depends also on $R$ (Marmorino and Caldwell).

The astrophysical relevance of thermohaline convection is to the edges of convective cores of stars (Spiegel), where the products of nuclear reactions, usually helium, take the place of salt. When the usual criterion for convective instability is employed in a massive stellar model evolving off the main sequence, it is found that once a sufficient, stable discontinuity of composition is built up at the edge of the convective core, the envelope immediately outside it is also convectively unstable. This has been considered unacceptable by many astrophysicists, and it is assumed that the discontinuity is somehow smoothed out, usually to precisely the degree that results in no more than marginal stability immediately beyond the truly convective core (Ledoux, Tayler, Schwarzschild and Härm), though other amounts of mixing have been proposed (Gabriel, Saio). Different criteria are used to define marginal stability, which lead to rather different results, but it does not seem possible to choose between them by astronomical means (e.g. Varshavskii; Sreenivasan and Ziebarth; Stothers and Chin; Robertson, Ziötkowski). This situation has some similarities to thermohaline convection set up by heating from below an initially isothermal layer of water stably stratified with salt, but the analogy is not perfect. Gabriel has argued against generalizing from laboratory experience in this case. However, the idea that at least one shell of ordinary convection is created outside the convective core does not seem implausible, though it is not obvious whether the interface separating the two convecting regions would be stable enough to survive the disrupting forces of the turbulence. Such a possibility has been pointed out by Tayler as a mathematically consistent alternative to the conventional procedure. To determine the structure of the region an understanding of the diffusive interfaces is required. Had $\Phi$ been independent of $\lambda$ and $R$ one might have had some confidence in extrapolating laboratory measurements, especially since, if thin convective layers are formed, this is one place where the Boussinesq approximation might be valid; but it appears that the answer is still out of reach.

Subcritical convection may also be relevant to solar type stars. It has been pointed out that the stability characteristics of the solar core are potentially similar to those of the thermohaline situation: overstable to infinitesimal perturbations and able to sustain direct convection of finite amplitude (Dilke and Gough). An important difference, however, is that whereas the usual saline layer derives its energy from an externally imposed heat source and will convect so long as that source is maintained, the sun must derive its extra energy from burning a supply of

${ }^{3} \mathrm{He}$ which is mixed from the edge of the core. The amount of ${ }^{3} \mathrm{He}$ available is finite and after it is burnt convection is presumed to cease, and the solar core becomes quiescent again until a new supply of fuel has accumulated near its edge. If it occurs, this process may have some bearing on the solar neutrino problem and the occurrence of terrestrial ice ages. Subsequent more detailed analysis has supported the overstability postulate (e.g. Noels et al., Unno), though some computations have cast doubt on it (Christensen-Dalsgaard and Gough). The likelihood of subcritical convection is questionable too (Ezer and Cameron; Ulrich), though some evidence for it has been found (Rood).

\section{Rotation and Magnetic Fields}

Uniform rotation inhibits convective motion and so increases the critical Rayleigh number above which convection can take place. At finite amplitude the motion can redistribute the angular momentum so that subcritical convection can occur (Veronis). Typically the constraint cannot be cancelled entirely and the rotation reduces the heat flux. This is not always true, however. Rossby found in the laboratory that rotation sometimes increases $N$ at fixed $R$, a behaviour seen also in three-dimensional numerical experiments (Somerville and Lipps) and a modal analysis (Baker and Spiegel). It gives fair warning to those who argue that factors inhibiting linear instability necessarily inhibit subsequent nonlinear development.

Astrophysical interest in the interaction between convection and rotation has been concerned in recent years with the structure of the convection zone in the solar envelope and the maintenance of the differential rotation. Of particular interest are the numerical experiments 
by Gilman. The subject has been reviewed recently by Gilman, Durney and Weiss (IAU Symposium 71). Tayler has discussed convection in rotating stellar cores.

The solar convection zone will not be understood until it is known how convection interacts with magnetic fields. It is hard to infer the field strengths beneath the surface, especially since the topology of the convective motion is such as to submerge the field (Drobyshevski and Yuferev). The formation and decay of magnetic field concentrations are of obvious interest, and are reviewed in the proceedings of IAU Symposium No 71. Like uniform rotation, a uniform magnetic field tends to inhibit convective motion. The linear stability characteristics of a plane Boussinesq fluid layer heated from below are similar to the rotating case with no magnetic field. But Weiss has pointed out that there are fundamental differences between Lorentz and Coriolis forces and that care must be taken when comparing the two cases. Weiss found that the nonlinear development of both overstable and direct infinitesimal motions can be oscillatory, provided the magnetic field is not too weak. The final state is not necessarily one in which there is equipartition between kinetic and magnetic energies (Peckover and Weiss). Modal calculations (van der Borght, Murphy and Spiegel) have revealed only a decrease in $N$ at fixed $R$ as the magnetic field increases, but a magnetic field appears to be able to interact with a rotating fluid in such a way that the resulting Nusselt number is greater than it would have been in the absence of the field (van der Borght and Murphy).

Of interest recently has been the question of whether convection can be the source of dynamo action. Childress and Soward demonstrated that the kind of flow encountered in a rotating convecting fluid is suitable for amplifying magnetic fields, as has been noticed also by Spiegel. Perturbation expansions about the marginal state (Soward; Roberts and Stewartson) and a modal analysis (Baker), both of which incorporate the forces on the fluid arising from the perturbed magnetic field, indicate that a convecting fluid can indeed sustain a magnetic field by induction.

\section{E. Time-dependent Convection}

New difficulties are encountered when a star is varying globally on a time-scale comparable with the convective turnover time. This may occur when the star is not in hydrostatic equilibrium : during gravitational collapse, a nova or supernova explosion, a flare or envelope ejection, or whenever a star pulsates. It is perhaps for pulsating stars that an understanding of the time dependence of convection is most urgently needed, because both theory and observations have progressed further than in the studies of other classes of intrinsically variable stars. Many of the gross features of the observations have been explained, but the position of the red edgelof the Cepheid strip, for example, remains unsolved. This can probably be blamed on an inadequate treatment of convection in the theoretical models.

Most computations of stellar pulsations have either ignored convection entirely, ignored perturbations (either Lagrangian or Eulerian) in the convective heat flux induced by the pulsations, | assumed the convection to adjust instantaneously to its changing environment, or assumed it to relax towards the state given by the usual mixing length formulae at a rate proportional to the amount by which it deviates from that state and inversely proportional to the eddy lifetime. The last of these prescriptions is perhaps the most credible, and was first used by Cox et al. to compute model Cepheids. However, its obvious deficiency is that it contains a free parameter: a constant of proportionality that determines the rate at which convection readjusts to the pulsation. This in turn determines the phase difference between the convection and pulsation, upon which the pulsational stability of the star directly depends.

Attempts have been made by Gough and Unno to generalize the mixing length theory. Unfortunately there are different ways of formulating the fundamental postulates. The resulting formulae are essentially the same for hydrostatic stars but differ when the star is presumed to pulsate. Moreover, there appear to be no relevant laboratory experiments with which to compare the various possibilities. Despite these uncertainties it would be interesting to know how sensitive pulsating stellar models are to the assumptions behind the convection formalism, and whether a possible choice of the uncertain parameters exists that rationalizes the observations. Computations in the quasiadiabatic approximation suggest this may be so, but apart from 
misrepresenting nonadiabatic effects these computations are deficient in an important respect: they do not take due account of the turbulent Reynolds stress.

It is usual to ignore the Reynolds stress when computing stellar models, partly because $a$ posteriori mixing length estimates are less than the gas pressure gradient in all but a thin region at the top of the hydrogen ionization zone. Attempts to include this stress in nonpulsating stars have been made, notably by Henyey et al. and Parsons but the formulation adopted is not entirely consistent. The mixing length formula for the Reynolds stress adds second derivatives of temperature and pressure to the hydrostatic equation, raising its order and introducing singular points at the edges of the convection zones. This has led to numerical instabilities which have been removed by judiciously ignoring high derivatives. It is claimed that this should not alter the results substantially. A consistent computation should be done to check.

It is even more important to include the Reynolds stress in pulsating models. The motion of most of the star is almost adiabatic; density and pressure perturbations are almost in phase and the work done in a single cycle is much less than the energy exchanged between thermal, gravitational and kinetic forms. There is no reason to suppose that the turbulent stress is in phase with the density, however, so even though its magnitude may be much less than the gas pressure gradient the work it does might not be negligible.

The modal approach adopted by Latour et al. includes the Reynolds stress. Because viscosity is included the equations are not singular, but the low Prandtl number gives such severe numerical trouble that it has not yet been possible to compute stellar models with deep convection zones. In principle this method can be used for pulsating stars, but certain aspects of the turbulent energy transfer are lost in the modal truncation and once again the results would have to be treated with some caution.

A recently discovered pulsating star of some interest is the sun (Hill and Stebbins; Fossat and Ricort; Severny, Kotov and Tsap; Brookes, Isaak and van der Raay), which is pulsating in many modes simultaneously. The pulsations are of too low an amplitude to have a noticeable influence on the structure of the star, but they could provide a powerful diagnostic tool. The oscillation periods are in satisfactory agreement with theoretical estimates (ChristensenDalsgaard and Gough; Scuflaire, Gabriel, Noels and Boury), but how the oscillations are driven is not yet known. It is unlikely that convection is unimportant. Differences between linear analyses which have either ignored convective flux perturbations (e.g. Shibahashi et al.) or have taken them into account (Noels et al.) using Unno's approach suggest that the stability of the modes of oscillation are rather sensitive to the assumptions adopted. In the light of experience with stars in hydrostatic equilibrium (Gough and Weiss) perhaps it is too optimistic to hope for an unambiguous solar calibration of time-dependent convection in the near future.

\section{THE Am AND Ap STARS}

(L. Mestel)

(Much of the material summarised here was presented at IAU Colloquium 32, 'Physics of Ap Stars', and at IAU Symposium 71, 'Basic Mechanisms of Solar Activity', 1975).

\section{A. Recent Observational Results}

Wolff and Wolff have presented evidence for two essentially continuous sequences of peculiar stars with main sequence luminosities, and temperatures that range from $7000^{\circ} \mathrm{K}$ to at least $20,000^{\circ} \mathrm{K}$. The first sequence consists of the $\mathrm{Si}-\mathrm{Cr}$-Eu-Sr stars which typically have observable magnetic fields and show spectrum and photometric variations. The second sequence is composed of peculiar stars without detectable magnetic fields, and includes, in order of increasing temperature, Am stars, hot Am stars, Hg-Mn stars, He-weak stars, and probably He-rich stars. It seems probable that the presence or absence of a magnetic field may well prove the distinguishing factor, but it has not been established that all $\mathrm{Si}-\mathrm{Cr}$-Eu-Sr stars have observable magnetic fields. Anomalous compositions are quite common among slowly rotating stars in this temperature range: there appear to be no normal A5-A9 stars with rotational 
velocities less than $40 \mathrm{~km} \mathrm{~s}^{-1}$, but there are at least some normal stars with Vsini $<100 \mathrm{~km} \mathrm{~s}^{-1}$. It is not clear whether anomalies occur in all slowly rotating early-type stars during part of their main sequence lifetime.

Babu's estimates of effective temperatures of Ap and Am stars indicate that they do not differ much from normal A star temperatures.

Most workers accept that the abundance peculiarities in magnetic stars are genuine. However, Steinitz and Gebbie argue that strong non-LTE effects in stellar atmospheres with magnetic fields can yield erroneous abundances if the usual curve-of-growth analysis is applied; they claim that the peculiarities ascribed to Ap stars could be spurious.

Landstreet and colleagues have applied to Balmer line wings a photoelectric technique similar to that employed in the solar magnetograph. The accuracy of field measurements so obtained is independent of rotation velocity. A survey of the more rapidly rotating Ap stars (periods 4 days or less) shows that fields in excess of $10^{3}$ gauss are significantly less common than among slower rotators (a conclusion also reached by the Wolffs). Thus besides the gross anti-correlation between rapid rotation, and the appearance of the Ap-Am phenomena and of surface magnetic fields, there is a similar anti-correlation within the class of magnetic stars. Magnetic curves obtained for several known magnetic stars show general agreement with photographic measurements; the differences in detail may provide new information about surface distributions of magnetic fields.

The oblique rotator model retains its plausibility as a phenomenological description of the magnetic variables (though one should bear in mind models with non-axisymmetric magnetic fields but with the magnetic and rotation axes aligned - a structure similar to the solar sector field (Wilcox)). Preston had concluded from analysis of the observations that the angle of inclination $\beta$ between rotation and magnetic axes tended to be either near $90^{\circ}$ or near zero. Borra had argued that the use of Landstreet's decentred dipole model modifies this conclusion - Preston's small $\beta$ cases could in fact be cases with large $\beta$ and large decentring parameter. Landstreet's newer observations have confirmed the existence of at least one small $\beta$ case. Mihalas has interpreted the helium-line variations in 56 Arietis and $\alpha$ Centauri by a model with two symmetrically placed helium-rich caps at the poles of a diameter inclined at a large angle to the rotation axis, the diameter to be identified with the symmetry axis of a mixed poloidaltoroidal field.

The Jascheks have confirmed the earlier conclusion of Abt and colleagues and Preston that the oblique rotator model is consistent, in that the values of $\mathrm{V}$ inferred from the magnetic and spectral periods are larger than the spectroscopically-determined Vsini, with $\mathrm{V}$ usually low compared with normal Am stars, and a random orientation of axes. Stift argues against Preston's tentative estimate of a mean Ap star radius of twice the zero-age main sequence radius for an A star. A number of interesting statistics seem to be emerging on Ap stars in binaries. Whereas most Am stars are members of moderately close binary systems, Ap stars are less commonly binary members than normal A stars (Abt and Snowden, Jaschek), though this seems not to be the case for the Hg-Mn stars (Abt and Snowden, Aikman). The shortest period of a binary containing an Ap star is about 6 days, as compared with 2 days for normal A stars. The Jascheks find an excess of small-mass companions to Ap stars in binaries.

\section{B. Recent Theoretical Work}

There appears to be general agreement that slow rotation is a necessary condition for Am stars (and perhaps for the corresponding non-observably magnetic Ap stars). Synchronization of spin and orbital angular velocities in moderately close binaries will yield abnormally slowly rotating $A$ stars, but if the two stars are too close the same synchronization will yield rapid rotators, while if they are too far apart tidal synchronization will be too slow. The most promising model to explain the abundance anomalies is probably Michaud's diffusion through gravitational settling and selective radiation force, applied especially to the Am stars by the Vauclairs and Stickland. The slow rotation yields a slower meridian circulation, so giving time for separation of elements to occur. Sinking of helium is important in causing the HeII convective zone to disappear (Vauclair), and also in yielding a negative gradient of mean 
molecular weight $\mu$, which strongly inhibits circulation in radiative zones (Mestel, Milsom). A full theory must take into account weak turbulent diffusion (Schatzman, Baglin) and the possible presence of any weak magnetic fields that can be dynamically significant while still optically unobservable. The attractions of the diffusion model are both its successes to date and the scope it offers for predictions about the stars of higher temperature in the Wolffs' scheme.

A somewhat different philosophy is advocated by Böhm-Vitense, who argues that since slowly rotating stars are generally considered to be normal, it is more helpful to think of the Am stars as normal, and the rapidly rotating A stars as those whose 'peculiarities' have to be studied. She cites effects in rapid rotators such as latitude-dependent gravity and surface temperature, and rotational interference with convection causing possible disappearance of a chromosphere.

The restriction of the Ap and Am phenomena to some early-type stars is plausibly explained by the powerful deep subphotospheric convection zones in late-type stars, which would rapidly mix any surface peculiarities that try and develop. The magnetic activity in the sun is thought by most to be evidence of an oscillatory dynamo. O.C. Wilson has observed variable calcium activity in other late-type stars, sometimes finding clear evidence of cyclic behaviour with a period of years. The observed decline of calcium activity with age is understandable if the inferred magnetic fields depend on the stars' rotation rates, and so decrease as the stars are braked by magnetically-controlled stellar winds.

The 'fossil' theory postulates that stellar magnetic fields may sometimes be slowly-decaying relics either of the galactic field in the gas from which the star formed, or of a field built up by dynamo action in an earlier epoch (Schüssler), but now no longer dynamo-maintained. Such a primeval field is likely to be expelled from the bulk of a convective zone (unless its energy were much greater than that inferred for even the strongest magnetic stars). It is thus not surprising that we see no evidence of a strong large-scale field on the sun or other late-type stars (though this does not preclude the possibility of a field trapped within the solar radiative core). In an early-type star the weak outer convection zones are ineffective against a field of 100 gauss or more. However, Cowling's classical calculation of the long decay time of stellar fields assumes implicitly that the field is dynamically stable. It is difficult to demonstrate conclusively that a particular field structure is stable against any and every disturbance, but it appears (Wright, Markey and Tayler) that a necessary condition is for the field to have toroidal flux linking the poloidal loops. Such a structure is inevitable in a dynamo-built field, and may develop spontaneously if the field is initially purely poloidal. It would be of great importance to determine a precise upper limit to the amount of magnetic energy allowed by hydromagnetic stability considerations (whatever the topology of the field).

The anti-correlation between stellar rotation and strong surface fields (with associated peculiarities) must be fitted into the theory. A possible explanation is suggested by a series of studies on thermal effects in rotating early-type magnetic stars (Wright, Davies, Monaghan, Moss, Mestel). In a rapid rotator with a fairly weak total magnetic flux $F_{t}$ the EddingtonSweet circulation flows, with the field maintaining the rotation nearly uniform, but being otherwise dy namically insignificant; in fact it is the circulation which tends to concentrate the field into the high-density regions deep down, with only a little flux $F_{s}$ emerging from the surface, by virtue of finite resistivity. However, if $F_{t}$ is supposed to increase, the magnetic disturbance to the thermal field becomes significant. In the computed steady states the circulation speed tends to be reduced, first in the surface regions and ultimately through the whole star, while an increasing fraction $F_{s} / F_{t}$ emerges from the surface, as required by Landstreet's and the Wolffs' observations. (Similar ideas may be relevant respectively to magnetic and non-magnetic white dwarfs). Because of the magnetic suppression of the circulation, abundance peculiarities can persist (Strittmatter, Mestel): the spectrum variations are plausibly associated with patchiness maintained by the magnetic field.

The limit of these solutions describes a non-rotating magnetic star with a structure determined by the condition of almost exact radiative equilibrium outside the convective one. It is not clear that such a model would be thermally stable - when perturbed the star may go over into a state with circulation currents which would convect and distort the field. (An example of this is the 'magnetic buoyancy' of a toroidal loop, which in a sub-adiabatic zone is a thermal 
rather than a dynamical phenomenon, and so would have a much longer time-scale than in a convective zone). It may be that a strong magnetic structure that is stable over stellar lifetimes requires centrifugal forces to be comparable with magnetic. Gradients of $\mu$ will also tend to stabilize against thermally-driven motion.

Magnetic braking of one sort or another is presumably responsible for the low rotations of at least the non-binary magnetic stars. Accretion braking (Havnes, Mestel) is an attractive possibility. Incoming gas will be forced approximately to corotate with the star, once it has reached the 'Alfvenic surface', where the magnetic energy and the kinetic energy of inflow are equal. If the centrifugal force exceeds gravity at the Alfvenic surface, the gas will then flow outwards, so carrying away angular momentum; the process cuts itself off when the angular velocity reaches a critical value, which turns out to be typical for an Ap star. Braking by a stellar wind is less attractive. First, it is doubtful whether early-type stars generate coronas hot enough to drive a thermal wind (though radiation-driven diffusion may have some effect (Michaud)); and secondly, if the magnetic field has a given strength, the consequent exponential law does not have a natural cut-off. The more efficient braking by stronger fields may account for part of the Landstreet-Wolff anti-correlation.

Havnes and Conti argue for selective accretion as an important contribution to the creation of surface peculiarities. There seem to be difficulties with the details of their model, such as the use of a particle rather than a fluid treatment. Michaud argues that e.g. infalling rare earth particles trapped in the magnetosphere will in fact be driven outwards by radiative force. He concludes that accretion is a perturbation on the dominant diffusive process.

The magnetic torques that slow up a star's rotation will also in general have a component tending to cause the instantaneous axis of rotation to precess through the star (Mestel, Mestel and Selley). Qualitative arguments (supported by one detailed computation) suggest that the axis looks for the region where the surface magnetic field is strongest. Another process (Spitzer, Mestel and Takhar, Mestel and Wright) that also tends to alter the obliquity angle depends on the dissipation of the oscillatory, dynamically-driven motions that necessarily arise in an oblique rotator. The asymptotic obliquity angle then depends on the bulk properties of the magnetic field. Both these dynamical processes deserve more study, to see whether one or other can account for the observed statistical properties of the obliquity angle. Another line worth pursuing is the purely kinematic effect of slow but persistent surface circulation on magnetic fields with different initial obliquities.

Much of the attraction of the fossil theory derives from apparent difficulties in accounting for the observations without allowing the total magnetic flux possessed by a star to be within wide limits a free parameter, determined by the history of the star from its formation epoch. However the recent advances in dynamo theory which have given us a plethora of possible models (Krause, Steenbeck, Raedler, Deinzer, Stix, Roberts, Weiss, Lortz, Moffat et al) certainly encourage us to ask whether contemporary dynamo action is responsible also for maintaining the fields of the magnetic Ap stars. One can e.g. postulate that the rotation field within the convective core has the gradient required to generate a steady rather than an oscillatory (solar-type) field, and that some of the flux appears at the surface. Alternatively, a non-uniform rotation and a large-scale circulation in the radiative envelope may maintain a non-axisymmetric field (Krause). However, most dynamo models in the literature are kinematic - they assume certain driving motions, such as non-uniform rotation, rather than simultaneously accounting for them. For example, one would expect the maximum flux generated over a solar cycle to be some increasing function of angular velocity $\Omega$; and indeed for solar-type stars, estimates of magnetic braking, assuming an algebraic relation between the mean field strength and $\Omega$ (Spiegel, Skumanich), do help in understanding the rotation of the sun and similar stars in the Hyades and Pleiades. But a full physical theory should predict the non-uniform rotation required e.g. for the solar dynamo: it is not yet clear that this has an essentially hydrodynamic explanation (Durney, Roxburgh, Gilman), or whether magnetic torques play an essential role. For non-oscillatory dynamos, one would expect the magnetic field to grow until the back-reaction of the magnetic stresses on some vital part of driving motion - e.g. the differential rotation - presumably limits the growth. A dynamo model for Ap stars that depends on non-uniform rotation and slow laminar circulation in the radiative 
envelope would probably have difficulty in generating fields of the observed strength. If instead we suppose that a steady dynamo is working in the convective core, and we see its manifestation at the surface, there remains the problem of accounting for the anti-correlation between observable field and rotation, both as between magnetic $A p$ and normal $A$ stars, and within the class of magnetic stars themselves. A possible answer may lie in a combination of dynamical dynamo theory and the work (summarised above) on the concentration of flux deep down by circulation. If the dynamo-built field has a total flux $F_{t}$ which varies like $\mid \Omega^{\mathbf{n}}$, and with $n>1$, then the observable flux should increase with rotation, contrary to observation; but if $0<\mathrm{n}<1$, then the increase in the total flux with $\Omega$ is more than off-set by the concentration of flux, so $F_{\mathrm{s}}$ may actually decrease with increasing $\Omega$ (Mestel and Moss). Even so there would remain serious difficulties. Why are there then non-magnetic slow rotators? In spite of the statistical correlations, there are anomalies, such as otherwise similar magnetic Ap stars with widely differing periods. Prima facie, these facts seem harder to fit into a contemporary dynamo theory than into a fossil theory.

Dolginov has revived the 'battery' process for generating Ap star fields. A non-spherical but axisymmetric helium distribution causes differences in the directions of the electron pressure and density gradients, yielding a net 'electromotive force' which slowly builds up a toroidal field. Meridian circulation based on an axis different from that of the helium distribution will generate a poloidal field from the toroidal. However, it is not clear that the process can start unless there is already a field present strong enough to prevent the non-spherical $\mu$-distribution from reverting to spherical symmetry.

\section{DYNAMICAL NUCLEOSYNTHESIS (W. D. Arnett)}

In this survey 'Dynamical Nucleosynthesis' is taken to refer to thermonuclear processes which synthesize nuclei in non-hydrostatic environments. Progress in this area is represented principally by (1) further analysis of the standard 'explosive' processes, (2) examination of nucleosynthesis in more realistic (or at least less abstract) environments, and (3) improved empirical knowledge of reaction rates.

\section{A. Processes}

It is useful to consider a nucleosynthesis process in a fairly abstract way, with a minimum number of parameters, to ascertain what constraints observed abundances place on the sites of nucleosynthesis. Pardo et al. have pursued this course in detail for carbon burning, and find the required peak temperature, density, neutron excess and expansion time scale are consistent with those expected from models of massive stars. Hainebach $e t$ al. have examined the e-process in similar detail. As a first attempt to explore the homogeneity of sites of this process, they examine optimum fits to solar system abundances using mixtures of varying neutron excess. This might correspond to matter processed at high and low densities for example. The best fit

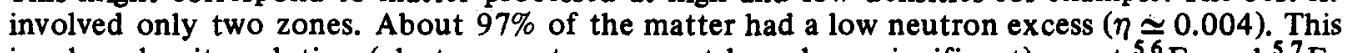
is a low density solution (electron-capture cannot have been significant); most ${ }^{56} \mathrm{Fe}$ and ${ }^{57} \mathrm{Fe}$ are made as ${ }^{56} \mathrm{Ni}$ and ${ }^{57} \mathrm{Ni}$. Only $3 \%$ of the matter had a high neutron excess $(\eta \simeq 0.077$ ), i.e., was processed at high density $\left(\rho>10^{9} \mathrm{~g} \mathrm{~cm}^{-3}\right)$ ! Cassé and Soutoul have noted that because of the finite half-lives of some of these nuclei, they might be detected in the cosmic rays as the unstable parent, giving a measure of the time between synthesis and acceleration. Clayton has worked out the implications for $\gamma$-ray astronomy of the synthesis of unstable ${ }^{22} \mathrm{Na}$ and ${ }^{57} \mathrm{Co}$; detection of their lines would probe the explosive process itself.

Blake and Schramm have examined the observational consequences of recently $\left(t<10^{9}\right.$ years) synthesized r-process material, and discuss what might be seen if such material were injected into the cosmic rays or were to appear on a stellar surface. Kritchman et al. have examined the (small) effect on r-process predictions of abundances caused by using a Garveytype mass law rather than a conventional one. Bisnovatyi-Kogan and Chechetkin have given an 
overview of what they expect to be the character of nucleosynthesis in supernovae and of the chemical composition in neutron star envelopes.

The most recent work on explosive burning in hydrogen and helium zones, and the 'Hot $\mathrm{CNO}$ cycle' is that of Arnould and Beelen who provide a detailed survey of possibilities as well as references to earlier work. Norgaard and Arnould (1975) have reanalyzed the expected nucleosynthesis from an exploding supermassive object (Audouze and Fricke). They used newer reaction data as well as including more nuclei with $A<12$, but no clear conclusions were possible.

\section{B. Environments}

\section{Novae}

In an interesting series of papers Starrfield, Sparks and Truran (SST) have constructed hydrodynamic models of a nova outburst, following the CNO abundances with a realistic nuclear reaction network. They find that they need enhanced CNO abundances to get a proper explosion, that ${ }^{15} \mathrm{~N}$ becomes the most abundant CNO nucleus, and that the ejection of mass comes from energy derived from $\beta^{+}$decay. The explosion around a $0.5 M_{6}$ white dwarf was less intense than for one of $1.0 \mathrm{M}$. Hoyle and Clayton suggested that in such an environment, with $\mathrm{H}$ and ${ }^{12} \mathrm{C}$ accreting until a thermonuclear runaway of $(p, \gamma)$ reactions occurs, the $s$ - and $r$-process might operate. Here ${ }^{13} \mathrm{C}(\alpha, n)^{16} \mathrm{O}$ provides neutrons. Starrfield et al. showed that this could happen, although with somewhat different parameters than in the Hoyle-Clayton picture.

\section{Supernova Shocks}

Colgate, Fowler and Hoyle have suggested that deuterium might be synthesized by spallation as a supernova shock traversed the outer, low density, parts of the star. Such light element formation depends on the existence of a high temperature precursor, which is a point of debate (Colgate; Weaver and Chapline). Epstein, Arnett and Schramm have shown that even if such a precursor does exist it is only possible to produce $\mathrm{D}$ without over-producing ${ }^{7} \mathrm{Li}$ and ${ }^{11} \mathrm{~B}$ in a high energy environment with few CNO nuclei. This is because ${ }^{7} \mathrm{Li}$ and ${ }^{11} \mathrm{~B}$ are readily produced by spallation on the CNO nuclei. The result is a general one; most of the deuterium we observe cannot have been produced by high energy reactions in Population I material.

Rather than assuming statistical equilibrium of nuclei, Bruenn and Marroquin have used a small reaction network to examine the structure of detonation waves in dense stellar material. Arnett had used a small reaction network coupled to a numerical hydrodynamic code, but insufficient computer time was available to explore more than one case.

\section{Reaction Rates}

The empirical basis of nucleosynthesis theory is a knowledge of nuclear reaction rates. Considerable progress has been made in this area (but there remains much more to do!). Vlieks, Morgan and Blatt have measured six $\alpha$-induced reactions on iron-group nuclei and Roughton, $e t$ $a l$. have measured $(p, \gamma)$ on eleven nuclei. Whitmire and Davids, Howard et al. and Rios, Schweitzer and Anderson have extended our knowledge of reaction rates of interest in advanced nucleosynthesis. Equally important is the extensive work by Woosley, Holmes, Fowler and Zimmerman (in press) which systematizes our knowledge of the data, and through a Hauser-Feshbach formalism, allows prediction of unmeasured cross-sections. This is vital for unstable nuclei, or nuclei which are difficult to measure for other reasons, but which are astrophysically important.

Helium burning sets the initial composition for many interesting processes of dynamical nucleosynthesis. The rate of the $3 \alpha$ reaction is sensitive to the rate of radiative de-excitation of the $7.655 \mathrm{MeV}$ state of ${ }^{12} \mathrm{C}$. Chamberlin et al., Davids, Pardo and Obst, and Mak et al. have remeasured this rate (i.e., $\Gamma_{\gamma} / \Gamma$ ) and a revision in the older value is necessary.

Our knowledge of spallation cross sections is improving. Oberg et al., Laumer, Austin and 
Panggabean, and Panggabean, Austin and Laumer have recently improved our understanding of $\mathrm{LiBeB}$ production from light nuclei $\left({ }^{13} \mathrm{C},{ }^{16} \mathrm{O},{ }^{20} \mathrm{Ne}\right)$. This is of considerable assistance to theoreticians (e.g., Canal, Ramaty and Kozlovsky, Epstein $e t$ al.).

Finally, work by Davids, Austin and their collaborators is filling in the gaps in our knowledge of masses of proton-rich nuclei above iron. These nuclei dominate the $\alpha$-rich freeze-out made of the e-process and may be probes of deep layers of supernovae.

\section{THEORY OF NEUTRON MATTER AND THE STRUCTURE OF NEUTRON STARS \\ (D. Pines)}

\section{A. Introduction}

This report gives a brief review of some recent developments in the theory of neutron matter and neutron star structure, together with related observations on pulsating compact $\mathrm{X}$-ray sources. By combining theory and observation it seems likely that we shall arrive not only at an improved understanding of the structure of neutron stars, but also of the fundamental interaction between neutrons in dense matter. Many of the theoretical results described are taken from a recent paper (Pandharipande, Pines and Smith, Astrophys. J. 1976, hereafter referred to as PPS), and the interested reader is referred to that paper for further details. In view of the fact that an overview of our understanding of the physics of neutron stars and related observations on pulsars and compact X-ray sources, as of September, 1973, may be found in the Proceedings of the Sixteenth Solvay Congress on Physics (1974), the period covered by the present report is essentially that of September 1973 to September 1975.

During the past two years, improved treatments of interaction between neutrons in dense matter have been developed (Bethe and Johnson; Walecka; Pandharipande and Smith) which suggest that the equation of state of neutron star matter in the $10^{14}-10^{15} \mathrm{~g} \mathrm{~cm}^{-3}$ region is considerably stiffer than that calculated earlier using the Reid (R) potentials. PPS find that these harder equations of state give rise to significant corrections for the structure of heavy neutron stars, which are found to have both larger radii and thicker crusts than the $R$ stars. Such corrections are important in the calculation of the frequency of free precession of a neutron star as well as those stellar parameters which determine the frequency of starquakes induced by pulsar spindown.

On the observational side, recent results of direct significance for neutron star models are the determinations of the masses of two neutron stars: Her X-1, which Middleditch, Mast and Nelson find has a mass of $1.33 \pm 0.13 \mathrm{M}_{\odot}$ and Vela X-1, which Rappaport and McClintock report has a mass greater than $1.7 M_{\odot}$. The Vela X-1 mass determination is important because it provides observational confirmation of the proposed stiff equations of state; in a sense, the neutron star is functioning as a nuclear physics laboratory. The Her X-1 mass determination is important because if the $35^{\mathrm{d}}$ X-ray periodicity observed for this star originates in stellar wobble, then one has two independent handles on the structure and evolutionary history of this star. These enable one to determine the current reference oblateness of $\mathrm{Her} X-1$, and to place limits on both the critical strain angle and initial angular velocity of this star.

The present report focuses on the above two topics: the way in which the behavior of dense neutron matter determines both the maximum mass and structure of a neutron star, and the extent to which the use of concepts in neutron star 'geology' (starquake theory, stellar wobble, etc.) enables one to learn about neutron star structure from observation. Other recent developments in neutron star theory are described only briefly: recent reviews of a number of other aspects of theoretical work on neutron stars have been given by Baym and Pethick (Ann. Rev. Nucl. Sci. 1975) and Canuto (Ann. Rev. Astr. Astrophys. 1975).

\section{B. Neutron Matter Equations of State and Stellar Structure}

In describing the structure of a neutron star it is convenient to divide the star into the following distinct density regions: 
(i) The surface $\left(\rho \$ 10^{6} \mathrm{~g} \mathrm{~cm}^{-3}\right)$, a region in which the nature and equation of state of matter can be significantly influenced by the temperatures and magnetic fields typically found in neutron stars.

(ii) The outer crust $\left(10^{6} \mathrm{~g} \mathrm{~cm}^{-3} \leqslant \rho \$ 4.3 \times 10^{11} \mathrm{~g} \mathrm{~cm}^{-3}\right)$, a solid region in which one finds a Coulomb lattice of nuclei and a relativistic electron gas.

(iii) The inner crust $\left(4.3 \times 10^{11} \mathrm{~g} \mathrm{~cm}^{-3} \leqslant \rho \$(2-2.4) \times 10^{14} \mathrm{~g} \mathrm{~cm}^{-3}\right)$, which contains a lattice of neutron-rich nuclei which coexist with a superfluid neutron gas and an electron gas.

(iv) The neutron liquid $\left((2-2.4) \times 10^{14} \mathrm{~g} \mathrm{~cm}^{-3} \leqslant \rho \leqslant \rho_{\mathrm{c}}\right)$, which contains mainly superfluid neutrons, with a small admixture of superfluid protons and normal electrons.

(v) A core region (at $\rho>\rho_{c}$ ), which may exist in some stars, depending on whether conditions are favorable for the formation of a charged pion-condensed state, a neutron solid, or some other phase which is physically distinct from the neutron liquid.

The equation of state in the surface region has little influence on the structure of the star, while that in the outer crust can be obtained to a good degree of accuracy by using semi-empirical mass laws to estimate the binding energies of the neutron rich nuclei in the outer crust. The equation of state of the inner crust depends on both the binding energies of the neutron rich nuclei found there (for which the most detailed and plausible calculations are those of Negele and Vautherin) and on the energy of the neutron gas which coexists with these nuclei. At comparatively low densities $\left(\rho<10^{13} \mathrm{~g} \mathrm{~cm}^{-3}\right)$, the contribution from the neutron gas energy is less important than the nuclear energy, and is reasonably calculated using, say, the Reid potential; however for densities in the $10^{13}-10^{14} \mathrm{~g} \mathrm{~cm}^{-3}$ region, where the neutron gas energy dominates the inner crust energy/nucleon, PPS find that use of stiffer interaction models leads to significant corrections to the equation of state. The exact density at which the inner crust ends and the neutron liquid begins is difficult to calculate; PPS consider two possible values for this transition density $\left(2 \times 10^{14} \mathrm{~g} \mathrm{~cm}^{-3}\right.$ and $\left.2.4 \times 10^{14} \mathrm{~g} \mathrm{~cm}^{-3}\right)$ and use equations of state for pure neutron matter to describe the neutron liquid regime.

The key ingredient in producing an accurate equation of state for neutron stars is the equation of state for pure neutron matter which, in turn, depends both on the interaction model used to describe the neutron-neutron interaction in dense matter and on the accuracy of the dense matter calculations one carries out using a given model. PPS consider four different interaction models:

(1) The $\mathbf{R}$ model, based on the phenomenological Reid N-N potentials which fit laboratory scattering data.

(2) The BJ model, based on phenomenological potentials obtained by Bethe and Johnson (Nucl. Phys. A230, 1, 1974).

(3) The TI (tensor interaction) model, based on the assumption that the attraction between nucleons comes entirely from the higher order contributions of the pion exchange tensor interactions (Pandharipande and Smith, Nucl. Phys. A237, 507, 1975).

(4) The MF (mean field) model, based on the assumption that the attractions between nucleons may be described phenomenologically as resulting from the exchange of an effective scalar meson (Pandharipande and Smith, Phys. Lett., 1975).

$A^{\prime}$ discussion of the respective merits of these different models and the resulting dense matter calculations is given by PPS, who also consider the plausibility and influence on the neutron matter equation of state of the various phase transitions which have been suggested for dense neutron matter: a neutron gas-liquid transition at densities of $3 \times 10^{12} \mathrm{~g} \mathrm{~cm}^{-3}$ (Chin and Walecka); $\pi^{-}$and $\pi^{\circ}$ condensation, which could conceivably occur at densities as low as nuclear matter density (Migdal; Sawyer and Scalapino); a neutron liquid-solid transition which has likewise been proposed for densities of the order of nuclear matter density (Palmer, Tosatti, and Anderson); and a transition to an abnormal state of matter, which has been suggested for densities varying from twice nuclear matter density to $\rho \sim 10^{16} \mathrm{~g} \mathrm{~cm}^{-3}$ (Lee).

Pandharipande, Pines and Smith point out that of the four interaction potential models, only the TI and MF models possess the property that the attraction between nucleons decreases with increasing density, a property which appears to be a general characteristic of microscopic models based on field-theoretical calculations. They argue that both the neutron gas-liquid transition and the transition to abnormal nuclear matter are unlikely to occur in neu- 
tron stars, that charged pion condensation, if it occurs, will not have a significant ef fect on the equation of state, while the existence of a $\pi^{\circ}$ condensed solid, shown by Pandharipande and Smith (PS) to occur only with the TI model, likely represents the most promising model for a possible solid neutron core. The existence of a neutron liquid-solid phase transition and the density change which occurs at such a transition are sensitive to the differences between the liquid and solid neutron matter energies, both difficult to calculate. PPS therefore considered three versions of the TI model: in one, (TI), the phase transition occurs at $\sim 7.1 \times 10^{14} \mathrm{~g} \mathrm{~cm}^{-3}$, and is accompanied by a $25 \%$ change in density; in a second, (TI1), it is assumed that the liquid and solid energies are close to one another for a considerable region around the transition density (the density increase is then only 3\%); in a third, (TI2), it is found that lowering the liquid energy by some $7 \%$ leads to an increase of the transition density to $\sim 8 \times 10^{14} \mathrm{~g} \mathrm{~cm}^{-3}$.

PPS find that use of stiffer equations of state for neutron stars has the following consequences:

(1) The maximum mass of a neutron star lies in the range 2-2.5 $M_{\odot}$ for the TI and MF models, as compared to the 1.4-1.6 $M_{\odot}$ value found using the Reid potential.

(2) If neutron solidification occurs (and it is only possible on the TI model), it acts to reduce the maximum mass of a neutron star; where an appreciable increase in density takes place at the transition, it results in a sharp cut-off in the mass, so that only a narrow range of masses and baryon numbers are accessible to such solid core stars.

(3) Heavy neutron stars $\left(M Z 1.3 M_{\odot}\right)$ have both larger radii and thicker crusts than had been predicted using the $\mathrm{R}$ model.

Even the most massive TI and MF neutron stars are not intrinsically high density systems; the heaviest stars have central densities $\sim 1.5 \times 10^{15} \mathrm{~g} \mathrm{~cm}^{-3}$, only six times the density of nuclear matter. This means, on the one hand, that theorists have a far better chance of carrying out realistic calculations of their stellar properties while, on the other, it tends to render less likely the possibility that one will find, deep within the stellar interior, various exotic forms of dense matter.

\section{Neutron Stars as a Probe of Nuclear Interaction}

PPS find that the maximum mass of a neutron star is essentially determined by the pressure of neutron matter in the vicinity of $10^{15} \mathrm{~g} \mathrm{~cm}^{-3}$. This pressure varies from $4 \times 10^{34} \mathrm{dyne} \mathrm{cm}^{-2}$

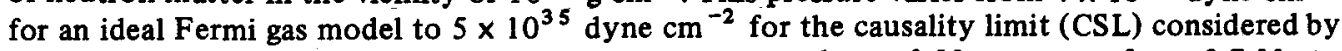
Malone, Johnson and Bethe, while the corresponding values of $M_{\max }$ range from $0.7 M_{\odot}$ to $3.02 M_{\odot}$. The latter limit is only slightly above that found by PPS for the MF model; the two equations of state (MF and CSL) resemble one another closely in the high density regime.

Rappaport and McClintock have observed the Doppler shift of the pulsed X-rays emitted by the binary X-ray source, Vela X-1; by combining their observations with optical measurements of the orbital velocity of HD 77581, the optical companion to Vela X-1, they have been able to determine the mass of Vela X-1. They conclude that its mass is $\geqslant 1.7 M_{\odot}$, based on a likely minimum value for the HD 77581 orbital velocity of $\sim 23 \mathrm{~km} \mathrm{~s}^{-1}$; the latter value is in agreement with that recently reported by van Paradijs et al. Since this mass is considerably in excess of the $1.4 M_{\odot}$ maximum mass of a carbon core degenerate dwarf, the identification of Vela $\mathrm{X}-1$ as a neutron star would seem inescapable. The maximum mass of an $\mathrm{R}$ star is $1.64 M_{\odot}$; if further observations of the orbital velocity of $\mathrm{HD} 77581$ confirm the minimum value of $23 \mathrm{~km} \mathrm{~s}^{-1}$, then one can rule out use of the Reid potential for a description of neutron interaction in dense matter solely on the basis of the Vela X-1-HD 77581 observations.

The present observational lower bound serves to rule out equations of state which are appreciably softer than that based on the $\mathrm{R}$ model; it provides considerable indirect evidence for the existence of a strong repulsive core in the neutron-neutron interaction, a conclusion which is quite compatible with our present understanding of nuclear-forces and the state-ofthe-art of calculations of the equation of state of dense matter. A mass determination of Vela X-1 which is less than $2 M_{\odot}$ is consistent with calculations based on either the TI or MF models considered by PPS. If, however, the mass of Vela X-1 (or some other neutron star) is found to exceed $2 M_{\odot}$, PPS find that this would imply a repulsion between nucleons which is 
much stronger than that suggested by high energy data on the $\omega$ meson-nucleon coupling. Such an observation would thus have significant implications for boson exchange models of nucleonnucleon interaction.

\section{Neutron Star 'Geology': Stellar Wobble and Starquakes}

Neutron stars, like the earth, possess a solid outer crust and a liquid interior; the more massive stars may possess a solid neutron core as well. It is interesting therefore to inquire whether geological and geophysical phenomena analogous to those which take place on earth can operate on a sufficiently global scale on a neutron star as to be visible from earth. Two obvious candidates for such global motions of a neutron star are starquakes and stellar wobble; these have been invoked in connection with the observed behavior of both binary X-ray sources and pulsars. The free precession, or wobble, of Her X-1 has been suggested by Brecher and Pines et al. as the origin of the $35^{\mathrm{d}}$ X-ray periodicity observed for that source (Giacconi), while quakes in the crust of the Crab pulsar (Ruderman, Baym and Pines) and in the hypothesized solid core of the Vela pulsar (Pines, Ruderman, and Shaham) have been suggested as the origin of the comparatively large and sudden spin-ups observed for these neutron stars (see Pines, Shaham, and Ruderman, IA U Symp. 531973 for a review).

The wobble frequency, $\Omega_{W}$, of a neutron star, reflects both its structure and evolutionary history; it is related to the rotation frequency by (Pines and Shaham)

$$
\frac{\Omega_{W}}{\Omega}=\frac{3}{2} \frac{B}{A+B} \epsilon_{0}
$$

Here $B$, the elastic energy coefficient, is $\sim \mu V_{c}$, where $\mu$ and $V_{c}$ are the shear modulus and volume of the solid portion of the star and $A$, the gravitational energy coefficient, is $\sim(3 / 25) G M^{2} / R$, the value appropriate to a self-gravitating incompressible sphere, $M$ and $R$ being the stellar mass and radius. $\epsilon_{0}$ is the reference oblateness, corresponding to that oblateness at which the crust or core of the star would be strain-free; it is determined by the angular velocity, $\Omega_{0}$, which the star possesses at the time the solid portion is form ed, and by the subsequent seismic history of the star. Consider, for example. a liquid core neutron star. At the time of crust formation, one has (Baym and Pines) $\epsilon_{0} \equiv \epsilon_{0}^{0}=I \Omega_{0}^{2} / 4 A$, where $I$ is the stellar moment of inertia. If $\epsilon_{0}^{0}$ is less than the critical strain angle, $\phi_{c}$, for crustal material, then, in view of the rapid neutrino cooling times and high crustal freezing temperatures, one would not expect appreciable plastic flow or oblateness-changing starquakes to occur for long after crustal formation; hence the current reference oblateness will be close to its initial value, $\epsilon_{0}^{0}$. If, on the other hand, $\epsilon_{0}^{0}>\phi_{c}$, one expects oblateness-reducing crustquakes to reduce the reference oblateness from its initial value, $\epsilon_{0}^{0}$, to some current value, $\epsilon_{0}$; the starquake condition is $\epsilon_{0}-\epsilon=\phi_{c}$, where $\epsilon$, the current stellar oblateness, is given by $I \Omega^{2} / 4(A+B)$.

For liquid core stars, $B \ll A$, while for solid neutron core stars, one may have $B \sim A$. Hence to the extent that one is able to place limits on $\phi_{c}$ or $\epsilon_{0}^{0}$, observation of free precession represents a promising approach to the detection of solid core neutron stars (Pines and Shaham). Moreover, for a liquid core star the parameters $A$ and $B$ turn out to be comparatively sensitive functions of stellar structure; hence for a given model equation of state one gets substantial variations in $A$ and $B$ on varying the mass of the star while, as shown in Table 1 , for a given stellar mass PPS find that the ratio $B / A$ is quite sensitive to the treatment of the neutron-neutron interaction.

The neutron star, Her X-1, is of special interest in any consideration of structure sensitive parameters: Middleditch et al. have determined its mass to be $1.33 \pm 0.13 M_{\odot}$; if one assumes that stellar wobble is the origin of the observed $35^{\mathrm{d}}$ X-ray periodicity, one can substitute the calculated values of $A$ and $B$ for a $1.33 M \odot$ star into (1) to obtain the reference oblateness of Her X-1; the resulting values are given in Table 1. As discussed by PPS, knowledge of this oblateness enables one either:

(i) to place a lower limit on the critical strain angle for the star $\left(\phi_{c} \gtrsim \epsilon_{0}\right)$ and determine the angular velocity the star possessed at the time of crustal formation, $\Omega_{0}=\Omega_{0}^{m i n} \equiv\left(4 A \epsilon_{0} / I\right)^{1 / 2}$; or 
(ii) if one assumes $\phi_{c} \lesssim \epsilon_{0}$, one can place a lower limit on the initial angular velocity $\left(\Omega_{0}>\Omega_{0}^{\min }\right)$, and determine the crustal critical strain angle $\left(\phi_{c} \simeq \epsilon_{0}\right.$, since $\left.\epsilon \ll \epsilon_{0}\right)$. The values of $\Omega_{0}^{m \text { tn }}$ determined in this way are likewise given in Table 1 .

Table 1. Stellar parameters for a $1.33 M_{\odot}$ Her X-1

$\begin{array}{lllllll}\text { Model } & \mathrm{I}_{4 \mathrm{~s}} & \mathrm{~B}_{45} & \mathrm{~A}_{\text {s2 }} & \mathrm{B} / \mathrm{A} & \epsilon_{0} & \Omega_{\text {min }}^{\text {[s] }} \\ \text { Reid } & 0.93 & 0.5 & 9.4 & 5.3 \times 10^{-6} & 5.2 \times 10^{-2} & 4600 \\ \text { BJ } & 1.33 & 3.4 & 7.2 & 4.7 \times 10^{-5} & 5.8 \times 10^{-3} & 1100 \\ \text { MF } & 1.97 & 6.7 & 5.4 & 1.2 \times 10^{-4} & 2.2 \times 10^{-3} & 490 \\ \text { TI } & 2.09 & 14.2 & 5.2 & 2.7 \times 10^{-4} & 1.0 \times 10^{-3} & 320\end{array}$

The values (or limits) which PPS find for $\phi_{c}$ and $\Omega_{0}$ using TI or MF stars appear to be reasonable ones; one sees, for example, that Her X-1 need not have been formed rotating much faster than has been hypothesized for the Crab pulsar using its current slowing down time as a guide. For $R$ stars, on the other hand, the inferred values of $\epsilon_{0}$ and $\Omega_{0}^{m i n}$ appear to be unreasonably large, so large indeed that Pines, Pethick and Lamb were led to suggest that Her X-1 might be a solid core neutron star. However, as discussed by PPS, the difficulties in calculating $A$ and $B$ for strongly inhomogeneous, thin crust, stars are sufficiently great that one should not perhaps rule out the Reid model solely on the basis of its inconsistency with the proposed wobble period of Her X-1.

It is, of course, not sufficient merely to hypothesize stellar wobble as a source of high-low $\mathrm{X}$-ray periodicity in binary X-ray sources; one must establish that the damping time for that wobble is sufficiently long, and the pumping mechanisms for it sufficiently efficient, that large amplitude wobble $(\sim 1 \mathrm{~km}$, say) can take place; otherwise, wobble, although it may exist, would not give rise to observable physical phenomena. To put the problem in a terrestrial perspective, eighty years after the discovery of the free precession of the earth by S. C. Chandler in 1893, the mechanisms responsible for damping and exciting the Chandler wobble (amplitude $\sim 6^{m}$ ) remain in doubt. The theoretical astrophysics group at the University of Illinois has devoted considerable attention to this problem in the past two years; they conclude that the wobble damping time due to crust-liquid friction is sufficiently long; that, to the extent that the flow of accreting matter is modulated by the wobble motion of the neutron star, in-phase torques exerted by accreting matter can excite large amplitude wobble for neutron stars in compact binary X-ray sources.

Some years ago Baym and Pines and later Pines and Shaham suggested that one could use the crustquake explanation for the sudden spin-up of the Crab pulsar to place limits on its mass. The argument was that the time between major crustquakes is of the order of the time required for pulsar spin-down to replenish the strain released in the previous quake; it was found that $t_{\mathrm{q}}(\Delta \epsilon) / T=\left(2 A^{2} / B I \Omega^{2}\right)|\Delta \epsilon|$, where $|\Delta \epsilon|$ is the magnitude of the change in stellar oblateness brought about by the hypothesized quake, and $T(\equiv \Omega / \Omega)$ is the spin-down time $(\simeq 2260$ y for the Crab pulsar). When this expression is applied to the September, 1969, Crab speed-up (Boynton et al.) for which $|\Delta \epsilon| \sim 9 \times 10^{-10}$, one finds that with the $\mathrm{R}$ equation of state the Crab pulsar would need have a mass $\$ 0.3 M_{\odot}$ in order that such crustquakes not be comparatively infrequent phenomena. This value posed difficulties both in evolving the neutron star and in explaining its overall luminosity $(L=I \Omega \dot{\Omega})$. On the other hand, using the TI equation of state, PPS find that only 10 years would be required for a Crab pulsar of mass comparable to that of Her X-1 to replenish the strain released in the September 1969, event. A Crab pulsar of this mass is thus quite compatible with elementary starquake theory; PPS then go on to consider possible structure sensitive parameters for the Crab pulsar, on the assumption that while its age and angular velocity are quite different from $\mathrm{Her} X-1$, its mass and structure may be quite similar. 


\section{E. Vortex Pinning in the Inner Crust}

Some time ago, it was shown by Pines that the vortices in that part of the rotating neutron superfluid which is located in the inner crust of the neutron star might be pinned to the nuclei in some crustal regions. Recently Anderson and Itoh have proposed that jumps in the pinned vorticity as a result of pulsar spin-down might explain the noisy behavior of the Crab, and possibly other pulsars (for an alternative explanation of the Crab behavior in terms of microquakes see Pines and Shaham). Ruderman has suggested that there may be still more dramatic consequences of this pinned vorticity; he argues that the Magnus force acting on the pinned vortices in a pulsar such as Vela will induce global strains of such magnitude that the subsequent crustquake will give rise to the 'giant' speed-ups observed for this pulsar. Anderson, Shaham and Pines have studied this question, and have tentatively come to the opposite conclusion. They find that the Magnus force is, in general, not as effective as the centrifugal force in inducing strain in the lattice; moreover, since it acts in the opposite direction, pinned vorticity will, under most circumstances, only impede the build up of strain energy in the crust by pulsar spin-down. It is further argued that in quakes, vortices, which are still strongly pinned to nuclei, are not likely to become unpinned to the extent that they would be capable of moving to their 'natural', unpinned sites; hence only a comparatively small frequency jump - a 'microglitch' - is likely to occur. There are a number of fascinating, and as yet not completely solved problems associated with the pinning, and subsequent unpinning of vorticity in the inner crust, so that this field of investigation is likely to remain active for some time to come.

\section{F. Tkachenko Modes in the Neutron Superfluid}

Tkachenko modes are oscillations of the vortex lattice which exists in a rotating neutron superfluid. They obey a sound-wave-like dispersion relation at low frequencies, with phase velocity in a neutron superfluid (Tkachenko, Ruderman) $c_{\mathrm{T}}=1 / 2\left(h \Omega / 2 m_{n}\right)^{1 / 2}$, where $m_{n}$ is the neutron mass. In a neutron star, the mode of lowest frequency, with a wavelength $\lambda$ comparable to the stellar radius, would have a period $P_{\mathrm{TK}} \sim 20\left(R / 10^{6} \mathrm{~cm}\right)(P / 1 \mathrm{sec})^{1 / 2}$ months, where $P$ is the rotational period of the star. Lamb, Shaham, and Pines have recently suggested that short time scale fluctuations in the torques acting on a neutron star which is accreting matter from a close binary companion may act to excite such Tkachenko modes, and that such excitation may explain the quasi-sinusoidal variation in pulse period observed for Cen X-3 and Her X-1 (Giacconi). As of this writing one cannot decide whether this interpretation of the observations, or one of the other interpretations (shot noise, slow but very large torque variations, or a triple star system) is correct; Lamb et al. discuss the way in which future observations will enable one to arrive at an unambiguous interpretation of the data.

\section{G. Stars with Solid Neutron Cores?}

Neutron solidification likely becomes possible only via the tensor force; moreover, it depends on a rather delicate balance between the liquid and solid energies over a comparatively wide range of densities. Thus if it can be shown to take place, it provides a test of the TI model, and rather detailed information concerning equations of state in the density regime, $6 \times 10^{14}<\rho<10^{15} \mathrm{~g} / \mathrm{cm}^{3}$. Possible candidates for solid core neutron stars include Vela X-1 (should its mass turn out to exceed, say, 1.8-1.9 $M_{\odot}$ ) and the Vela pulsar (PSR 0833-45). The latter possibility was suggested by Pines, Shaham and Ruderman as a way of explaining the two 'giant' speed-ups observed for this pulsar (Radhakrishnan and Machester, Reichley and Downs); they proposed that quakes in the hypothesized solid core of the star might give rise to speed-ups of the observed magnitude and frequency. There are problems with this explanation (e.g., the elastic energy release may be so large as to make the Vela pulsar an easily visible soft $\mathrm{X}$-ray source) and other explanations for the speed-ups have been proposed (e.g. Ruderman, Astrophys. J., 1975).

As Shaham and Pines have emphasized, detection of free precessional motion of the Vela pulsar would provide an unambiguous test of whether it has a.solid core; if it did, it would 
precess with a period $P_{W}=2 / 3\left(P_{R} / \epsilon_{0}\right)(A+B) / B \sim\left(P_{R} / \epsilon_{0}\right) \sim \min$, while its precessional period would be some three orders of magnitude longer if it is a liquid core neutron star. Indeed, the pulse periods of Vela X-1 $\left(283^{\circ}\right)$ and of several of the recently discovered pulsating transient $\mathrm{X}$-ray sources are of just the magnitude one would expect for the wobble period of a solid core neutron star which rotates with period $\geqslant 0.1^{\mathrm{s}}$. However, as PPS point out, in the absence of positive identification of a fast periodicity in these latter sources, any wobble interpretation of their periodicity would seem extremely speculative.

\section{H. Charged Pion Condensation}

There are two quantities of interest in connection with the question of pion condensation in dense neutron star matter; the threshold density at which such condensation occurs and the lowering of the system energy brought about by such condensation, assuming that it takes place. Detailed calculations of the $\pi$ condensation threshold density in pure neutron matter (Bäckman and Weise, Bertsch and Johnson) which include the s- and p-wave pion-nucleon interaction, coupling to the $\Delta$ resonance state, and the effects of nuclear forces suggest that the condensation is likely at $\rho>6 \times 10^{14} \mathrm{~g} \mathrm{~cm}^{-3}$; in this case it would affect only the more massive $\left(M>1.4 M_{\odot}\right)$ neutron stars. The change in the energy of matter due to $\pi^{-}$condensation has not yet been calculated to a similar degree of sophistication. PPS argue that the very significant effects suggested by Hartle, Sawyer and Scalapino, which result from a cancellation between the small positive matter energies predicted with the Reid interaction and the large negative pion condensation energies that they calculate, are unrealistic both because calculations based on the Reid potential give matter energies which are too small, and because it is doubtful that the condensation energy is as large as that which Hartle, Sawyer and Scalapino calculate; for example that estimated by $\mathrm{Au}$ and $\mathrm{Baym}$ is an order of magnitude smaller. Under these circumstances, the condensed pions would have little effect on neutron star structure, although they could play an important role in the cooling of neutron stars, as first suggested by Bahcall and Wolf.

\section{Acknowlegments}

It gives the author pleasure to thank his colleagues in the University of Illinois astrophysics group (Gordon Baym, I. Easson, D. Q. Lamb, F. K. Lamb, C. J. Pethick, V. R. Pandharipande, J. Shaham and Roger Smith) for stimulating conversations on these and related topics. The work of our group which is described in this report has been supported in part by the National Science Foundation. 